\title{
Preliminary results from the MEMO multicomponent measurements of waves on-board INTERBALL 2
}

\author{
F. Lefeuvre ${ }^{1}$, M. Parrot $^{1}$, J. L. Rauch $^{1}$, B. Poirier ${ }^{1}$, A. Masson ${ }^{1}$ and M. Mogilevsky ${ }^{2}$ \\ ${ }^{1}$ CNRS/LPCE 45071 Orléans cedex 02, France \\ ${ }^{2}$ IKI, Russian Academy of Sciences, 117081, Moscow, Russia
}

Received: 25 November 1997 / Revised: 29 May 1998 / Accepted: 3 June 1998

\begin{abstract}
The MEMO (MEsure Multicomposante des Ondes) experiment is a part of the INTERBALL 2 wave consortium. It is connected to a total of six electric and nine magnetic independent sensors. It provides waveforms associated with the measurement of two to five components in three frequency bands: ELF (5$1000 \mathrm{~Hz})$, VLF $(1-20 \mathrm{kHz})$, LF $(20-250 \mathrm{kHz})$. Preliminary analyses of low and high resolution data are presented. The emphasis is put on the estimation of the propagation characteristics of the observed waves.VLF hiss emissions are shown to be mainly whistler mode emissions, but other modes are present. An accurate estimation of the local plasma frequency is proposed when the low $L=0$ cutoff frequency is identified. AKR emissions observed just above source regions are studied. R-X and L-O modes are found: the first at the lowest frequencies and the second at the highest. Both propagate with wave normal directions weakly oblique or quasi-parallel to the Earth's magnetic field direction. Propagation characteristics are also determined for a (non-drifting) fine structure of AKR. There is no fundamental difference with structurless events. Nightside and dayside bursts of ELF electromagnetic emissions are presented. It is not clear whether the two emissions belong to the "lion roar" emissions or not.
\end{abstract}

Key words. Magnetospheric physics (auroral phenomena; plasma waves and instabilities; instruments and techniques)

\section{Introduction}

Although wave field measurements made on board satellites such as ISIS-1, ISEE- 1 and 2, DE 1, VIKING and $\mathrm{AKEBONO}$ have provided a lot of information on

Correspondence to: F. Lefeuvre the wave emissions present in the Earth's auroral regions, numerous questions are still open on the generation mechanisms as well as on the wave-wave and wave-particle interactions taking place in this very active zone. With the launch of the INTERBALL 2 spacecraft on September 6, 1996, on an elliptical orbit of inclination $65^{\circ}$, and of apogee and perigee at $19200 \mathrm{~km}$ and $772 \mathrm{~km}$, there is an opportunity to cross the interaction regions and to follow the same line of force over time intervals of the order of one hour or more. The POLAR and FAST satellites launched a few months before and after explore higher and lower altitudes respectively.

Among the wave emissions present in the auroral zones, the auroral kilometric radiation (AKR) is the most intense. Numerous papers have been devoted to the study of this phenomenon. In what follows, we shall mainly refer to the ones presenting sets of data comparable with those of INTERBALL 2 (Gurnett et al., 1979, 1983; Gurnett and Anderson, 1981; Calvert and Hashimoto, 1990; de Feraudy et al., 1987, 1988; Bahsen et al., 1989; Ungstrup et al., 1990; Roux et al., 1993) and to reports about temporal fine structures (Gurnett et al., 1979; Gurnett and Anderson, 1981; Benson et al., 1988) and fine narrow-bandwith drifting structures with rapidly varying center frequencies (Gurnett et al., 1979; Gurnett and Anderson, 1981; Morioka et al., 1981; Baumback and Calvert, 1987; Benson et al., 1988). First presentations of MEMO data also dealt with AKR harmonics. But, it has been discovered since, that MEMO AKR harmonics were due to a non-linear transform improperly applied to waveform data.

Most generation models of AKR are based on the cyclotron-maser mechanism of $\mathrm{Wu}$ and Lee (1979). However, any new wave and particle data generates new models (Potelette et al., 1992; Mourenas et al., 1993) or new versions of the existing models. VIKING measurements performed during source crossings have provided a lot of new insights (Hilgers et al., 1991, 1992; Roux et al., 1993 and references therein). We will show that INTERBALL 2 measurement just above source regions 
demands models with a confinement in the source regions (Calvert, 1981, 1982; Louarn and Le Quéau, 1996a, b).

Whistler mode auroral hiss and Z-mode, frequently observed in the auroral zone, have been well reviewed by Gurnett et al. (1983). They are presumed to be generated by instabilities related to low-energy auroral electrons. Broad band electrostatic noise (BEN) and extremely low frequency waves have also been observed (Pottelette et al., 1987; Bahnsen et al., 1989) and appear to be concentrated in the region of particle accelerations.

At present, most studies made on the auroral wave emissions are based on rough estimates of the propagation characteristics. They are obtained either from the values of high and low cutoff frequencies observed on spectrograms or from time variations of electric field measurements in the plane of rotation of the satellite (spin-null technique). More direct estimations of the sense of polarization of AKR are provided on DE 1 by correlating the signals obtained at the output of two orthogonal dipole antennas (Shawhan and Gurnett, 1982) or by using a spin-phase technique (Calvert, 1985). However, approximations are still needed. The only way to obtain non-ambiguous estimates of the full propagation characteristic is to perform a wave analysis on the waveforms associated with a minimum of four electromagnetic wave-field components: preferably, the three magnetic components and one electric component (Lefeuvre et al., 1981, 1982, 1986; Parrot et al., 1989). This has been done for ELF and VLF waves observed on AKEBONO (Kimura et al., 1990; Kasahara et al., 1995). However, the altitudes are generally too low to be compared with INTERBALL 2. As a matter of fact, waveforms in wide frequency bands are also required to test for the presence of wave-wave interactions and more generally to characterize turbulent phenomena (Lagoutte et al., 1989; Krasnosel'skikh and Lefeuvre, 1993; Kravtchenko-Berejnoi, 1994; Kravtchenko-Berejnoi et al., 1995a, b).

It is the aim of the MEMO experiment to transmit to the ground the waveforms of the signals associated with the measurement of four to five wave-field components (generally, three magnetic and one or two electric) in frequency bands from a few $\mathrm{Hz}$ to $250 \mathrm{kHz}$ (for a full description see Lefeuvre et al., 1998). According to the memory capacities available at the time of the conception of the instrument (1983) and the allocated bit rate (around $10 \mathrm{kbits} / \mathrm{s}$ ) this is possible in very short time intervals only. The main operational mode of MEMO is a snapshot mode. Preliminary results, presented here, show that, despite a functional degradation and a fatal failure four months after switching on, the MEMO experiment has fulfilled part of its initial objectives.

The plan is as follows. Section 2 deals first, with the MEMO equipment and the functioning mode, and second, with the MEMO data and the wave analysis techniques applied in the following sections. Section 3 is devoted to analyses of AKR emissions and associated phenomena (auroral hiss, density cavities, BEN, ELF emissions, etc.) just above source regions. AKR fine structures observed on MEMO are discussed in Sect. 4. Bursts of ELF electromagnetic emissions are briefly described in Sect. 5. Finally, Sect. 6 provides provisional conclusions.

\section{Instrument description}

\subsection{Sensors}

In order to provide a very comprehensive set of wave measurements in the frequency range $0.01 \mathrm{~Hz}-2 \mathrm{MHz}$, the MEMO experiment is associated with three other experiments: POLRAD, in the $\mathrm{LF} / \mathrm{MF}$ frequency range; NVK-ONCH, in the ELF (EBF) and VLF (TBF) frequency ranges; and, via NVK-ONCH, IESP, in the ULF $(f<30 \mathrm{~Hz})$ frequency range.

2.1.1 Electric sensors. The location and orientation of the various electric antennas are shown in Fig. 1, where the $\mathrm{X}$ axis is along the spin axis of the satellite, i.e. approximately in the Sun direction (reference). The Y and $\mathrm{Z}$ axis are perpendicular to each other and to the $\mathrm{X}$ axis and form the usual Cartesian co-ordinate system.

Very low and extremely low frequency electric fields $(0-20 \mathrm{kHz})$ measurements are provided by the IESP dipole antennas (Perraut et al., this issue). Each dipole consist of spherical (vitreous carbon) conductors of $8 \mathrm{~cm}$ diameter located at the end of insulated booms. The field components are denoted $E_{x}, E_{y}$ and $E_{z}$. For the $E_{x}$ component the distance between spheres is $4.31 \mathrm{~m}$. In order to avoid any shadow effect, the boom is tilted relative to the $\mathrm{X}$ axis. It is inclined at $7^{\circ} 5$ to the XY plane and $6^{\circ} 3$ to the XZ plane. For the $E_{y}$ and $E_{z}$ components the distance between the spheres is $22 \mathrm{~m}$.

Low and medium frequency electric fields $(40 \mathrm{kHz}-$ $2.2 \mathrm{MHz}$ ) are measured by the POLRAD experiment (Hanasz et al., this issue). Two $22 \mathrm{~m}$ tip to tip dipoles and a $5 \mathrm{~m}$ monopole boom electric antennas are used. The components are denoted $E_{1}, E_{2}$ and $E_{3}$. The 1 axis is along the spin axis of the satellite (X). The 2 and 3 axis are the $\mathrm{Y}$ and $\mathrm{Z}$ axis rotated by $45^{\circ}$. The $E_{1}$ component $\left(\mathrm{A}_{5}\right)$ is measured by the monopole antenna. The $E_{2}\left(\mathrm{~A}_{1}, \mathrm{~A}_{3}\right)$ and $E_{3}\left(\mathrm{~A}_{2}, \mathrm{~A}_{4}\right)$ components are measured by the dipole antennas. In order to avoid interference due to the $\mathrm{DC} / \mathrm{DC}$ converters, the measurements between 20 and $40 \mathrm{kHz}$ are not taken into account.

2.1.2 Magnetic sensors. Three sets of search-coils are necessary to cover the frequency band between $0.01 \mathrm{~Hz}$ and $2 \mathrm{MHz}$. They are labelled: ULF/ELF (or ULF/ EBF) below $1 \mathrm{kHz}, \mathrm{VLF}$ (or TBF) between 1 and $20 \mathrm{kHz}$, and $\mathrm{LF} / \mathrm{MF}$ between $40 \mathrm{kHz}$ and $2 \mathrm{MHz}$. Their location and orientation are indicated in Fig. 1. The ULF/ELF antennas are part of the MEMO 


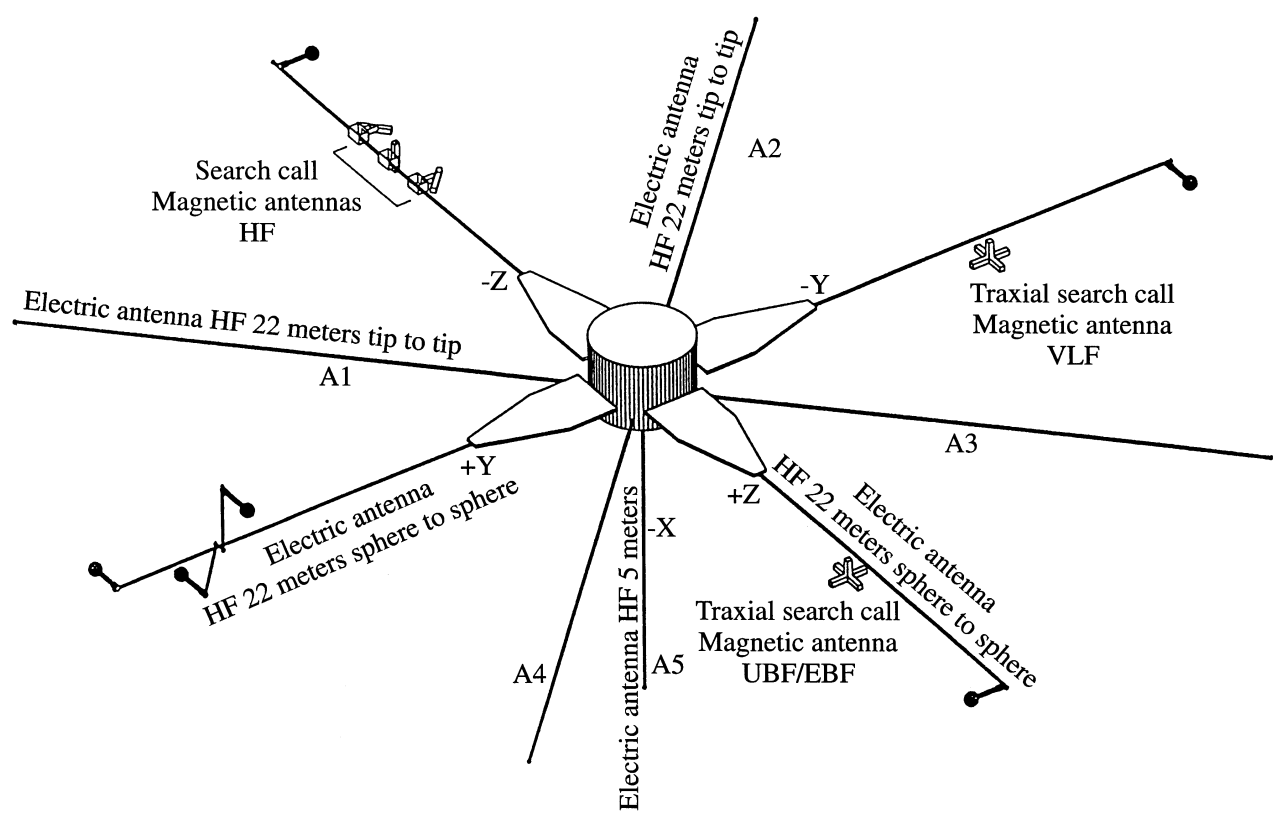

Fig. 1. Description of the electric and magnetic sensors on the spacecraft

experiment. They are the heritage of three previous space programs GEOS 1-2 (S-300 experimenters, 1979), ARAKS (Delahaye et al., 1978), and ARCAD 3 (Berthelier et al., 1982). The sensors consist of a $\mu$ metal core around which 80000 turns of wire are wound. They are aligned along the $\mathrm{X}, \mathrm{Y}$ and $\mathrm{Z}$ axis of the satellite. In what follows, they are denoted $\mathbf{B}_{x}, \mathbf{B}_{y}$, $\mathrm{B}_{z}$. The mechanical characteristics are given in Lefeuvre et al. (1998).

The VLF antennas are part of the NVK-ONCH experiment (Mogilevsky et al., this issue). They are similar to the ones flown on the ACTIVNY satellite. The sensors consist of a ferrite core around which approximately 100000 turns of wire are wound. They are aligned along the $\mathrm{X}, \mathrm{Y}$ and $\mathrm{Z}$ axis of the satellite. They are denoted $\mathrm{b}_{x}, \mathrm{~b}_{y}, \mathrm{~b}_{z}$.

The LF/MF antennas are part of the MEMO experiment. In order to avoid mutual induction, they are located at different places on the same boom with minimum and maximum separation distances of 42 and $90 \mathrm{~cm}$, respectively. They consist of ferrite cores around which approximately 100 turns of wire are wound. The sensors are aligned along the 1, 2 and 3 axis, and are denoted $\mathrm{B}_{1}, \mathrm{~B}_{2}, \mathrm{~B}_{3}$. The mechanical characteristics are given in Lefeuvre et al. (1998).

For the three sets of sensors, flux feedback systems enable the adjustment of the bandwidth to the desired specifications. Preamplifiers are used to increase the measured signals to a level where they can be analyzed on-board. The transfer functions and the sensitivity curves of the MEMO sensors, taken at the output of preamplifiers are given in Figs. $2(\mathrm{UBF} / \mathrm{EBF})$ and $3(\mathrm{LF})$ respectively. Differences among the $B_{1}, B_{2}$ and $B_{3}$ curves are due to differences in the length of the cables between the antennas and the preamplifiers. In the frequency of maximum interest for MEMO $(250 \mathrm{kHz})$, they are negligible.
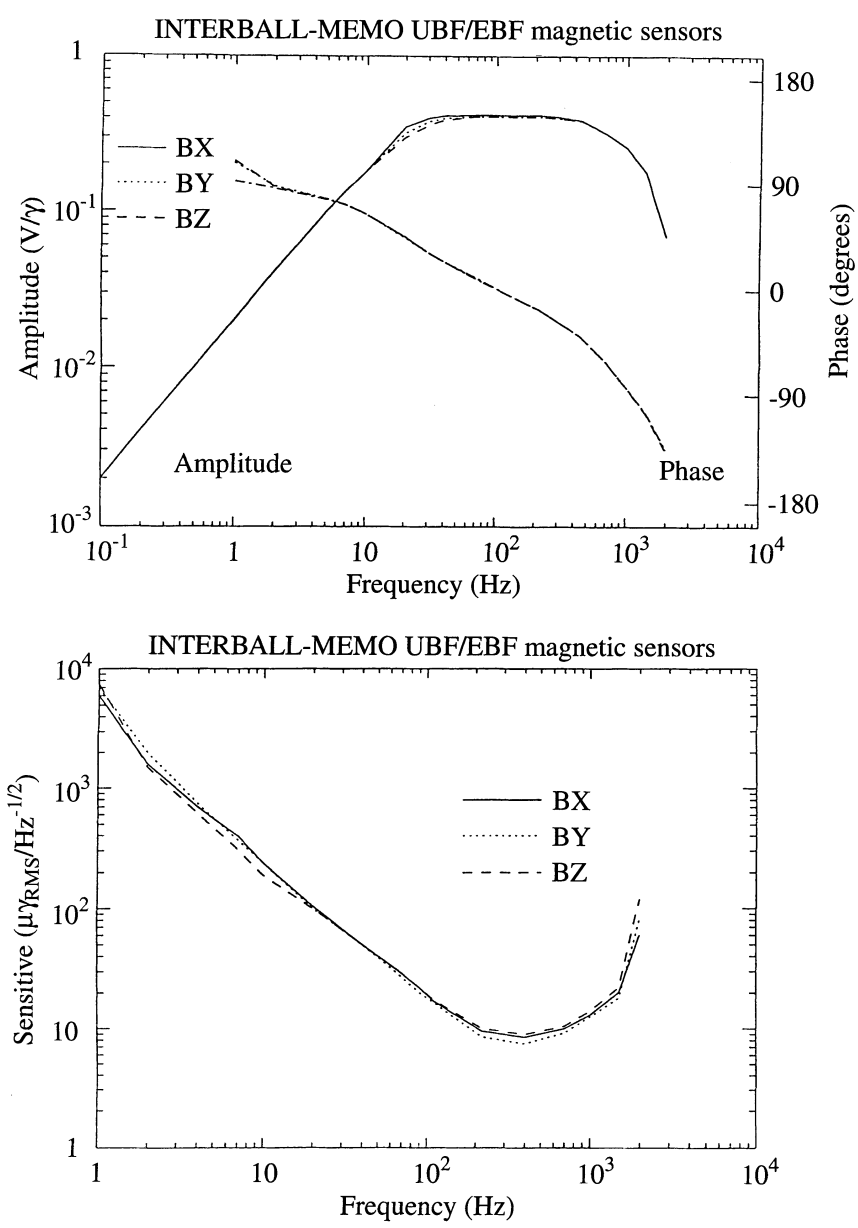

Fig. 2. Transfer functions and sensitivity curves of the UBF/EBF magnetic sensors 

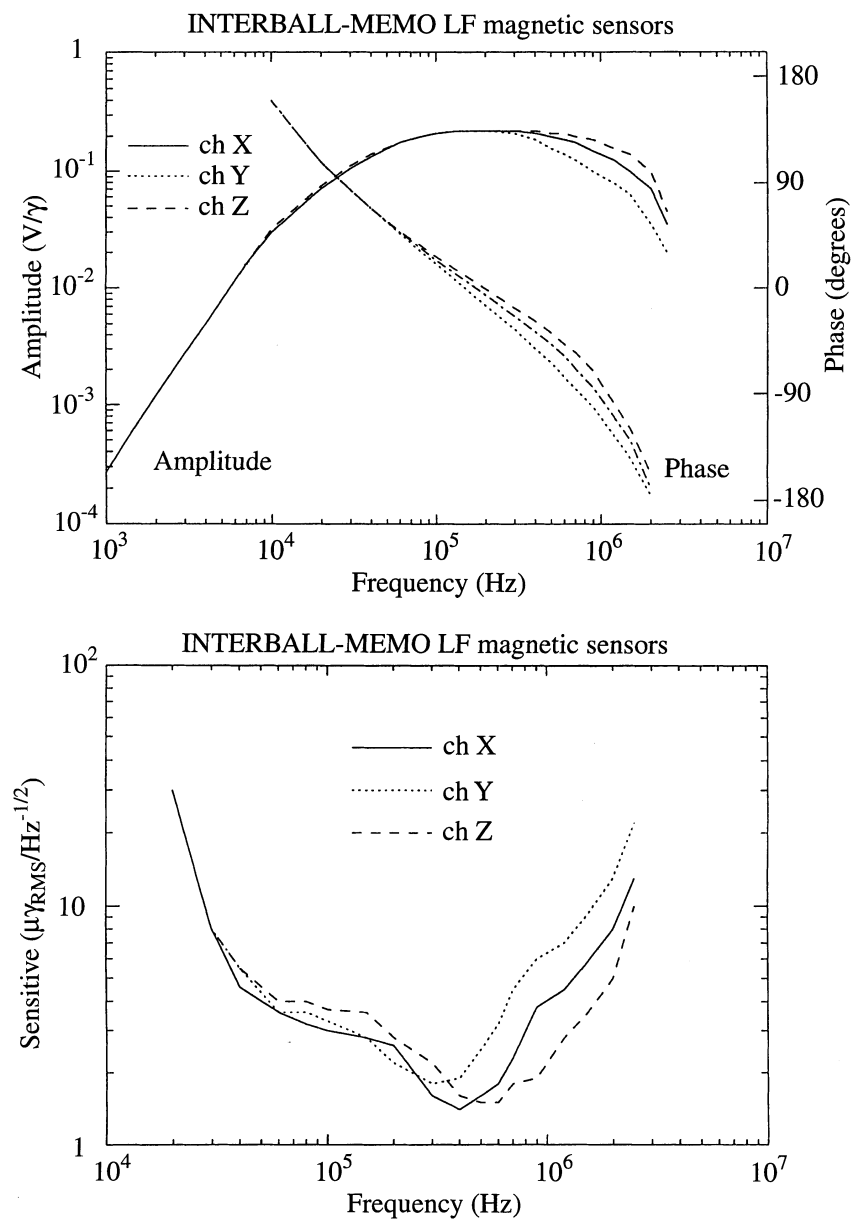

Fig. 3. Transfer functions and sensitivity curves of the LF magnetic sensors

\subsection{Electronic instrumentation}

2.2.1 The wave consortium. Information transactions in the wave consortium are quite complex. They can be summarized as follows. The three output signals from the preamplifiers of the magnetic sensors $B_{1}, B_{2}, B_{3}$ are transmitted to MEMO. A connection allows the transfer of the signal to POLRAD. The three output signals from the differential preamplifiers of the electric signals $E_{1}, E_{2}, E_{3}$ are transmitted to POLRAD where they can be attenuated by four step attenuators $(0,-20 \mathrm{~dB},-40$ $\mathrm{dB},-60 \mathrm{~dB})$. Another connection then sends them to MEMO. Within the electronic block of POLRAD, the three electric signals or the three magnetic signals are entered into step frequency analysers (SFAs) which place pass band filters of 4 or $30 \mathrm{kHz}$ bandwidth anywhere between $4 \mathrm{kHz}$ and $2.2 \mathrm{MHz}$. The output signals are sent to a polarimeter (for estimation of the Stokes parameters) and, when requested, to MEMO. During the time interval where the POLRAD relaxation sounder is $\mathrm{ON}$, one of the signals, sent to MEMO in a $4 \mathrm{kHz}$ bandwidth, is used to perform high resolution measurement of the electron density.

The power supplies of the low frequency electric sensors and magnetic sensors are within IESP and NVK-ONCH respectively, which means that the sensors are switched ON or OFF at the same time as those two experiments. The signals issued from the preamplifiers of the magnetic sensors are sent to NVK-ONCH. The signals that issue from the differential amplifiers of the electric sensors are sent to IESP. The electric signals are entered into a multiplexor which selects two channels: $E_{y}$ and $E_{x}$ or $E_{z}$.

The signals associated with the two electric channels plus those associated with the three ULF/ELF magnetic channels are passed into the extremely low frequency pass-band filters $(5 \mathrm{~Hz}-1000 \mathrm{~Hz})$. A connection then sends the five output signals to MEMO and two of them (one electric and one magnetic) to identical sets of 10 adjacent filters. In between, the two signals are amplified by a two step amplifier $(0,30 \mathrm{~dB})$. The outputs of the NVK-ONCH filter banks are connected to a telemetry system and to MEMO.

At the same time, the signals associated with the same two electric channels plus the signals associated with the three VLF magnetic channels are passed into low frequency pass-band filters $(1-20 \mathrm{kHz})$. The five outputs are sent both to MEMO and, after amplification, to step frequency analysers (SFAs). The SFAs allow insertion of pass-band filters of $800 \mathrm{~Hz}$ bandwidth anywhere in the range $1-20 \mathrm{kHz}$, in steps of $500 \mathrm{~Hz}$. Upon request, the filtered signals may be stored by MEMO.

Logical operations allow exchanges of commands, technological parameters and status between all wave experiments. They are used for common modes of operation (in flight calibrations, recording of POLRAD or NVK-ONCH data within MEMO, triggering of data collection, etc.).

2.2.2 The MEMO memory systems. All signals entered into MEMO are filtered and then amplified by three step amplifiers before being digitized into 8-bit words. The amplifier gains are 12, 24, $36 \mathrm{~dB}$ for the LF signals, -6 , +6 and $18 \mathrm{~dB}$ for the VLF and ELF signals. There is an exception for the SFA signals which have a two step amplifier: -9 and $+6 \mathrm{~dB}$. Filter parameters and gain factors are given in Lefeuvre et al. (1998).

According to the operational modes that are controlled by the microprocessor (here a NSC 800), several digitized waveforms are sent to each of the two memory systems. These are a small memory system MEDOR (512 8-bit words) and a relatively large direct memory access (DMA) system (786 000 8-bit words).

Two (occasionally four) digitized waveforms are systematicaly sent to the MEDOR system. A fixed number of samples (generally 256, exceptionally 128) of each waveform is transmitted by the telemetry system. Basically, the time interval between two runs is $0.2 \mathrm{~s}$ for a direct telemetry (TM) system named STO (a faster mode is forecast, but it assumes that the bit rate increased from $20 \mathrm{kbits} / \mathrm{s}$ to $40 \mathrm{kbits} / \mathrm{s}$ ), and $0.5 \mathrm{~s}$ for a memory TM system named SSNI. MEMO cannot be connected to two different telemetry systems at the same time. Because of the relatively short time interval between two data collections of 256 or 128 samples, the MEDOR data become low-resolution data. 
Two to five digitized waveforms are sent to the DMA system. For each waveform, blocks of $N$ successive samples (with $N$ varying from 128 to 171000 ) are either periodically recorded (with time intervals of 0.2 to $64 \mathrm{~s}$ ) or recorded after a POLRAD flag or a NVK-ONCH flag has been received. The DMA data are essentially high-resolution data. However in some MEMO modes, the DMA system is used to produce low-resolution data. In that case, the time intervals between two sets of 256 or 128 samples are given by one of the values that are imposed on MEDOR by the telemetry system.

All MEMO data are formatted by the NSC 800 microprocessor. The MEDOR data are periodically sent to the telemetry system. The amplitude levels of the DMA data are tested before they are transmitted by the telemetry system.

A major limitation of the system is that data cannot be recorded while the DMA data are being transmitted to the telemetry system. This produces large gaps in the measurements. For instance, as MEMO generally uses half the bit rates of the STO system, the gap is of the order of $5 \mathrm{~min}$ when STO is working at $40 \mathrm{kbits} / \mathrm{s}$ (which is a maximum) and 10 min when STO is working at $20 \mathrm{kbits} / \mathrm{s}$.

\subsection{The MEMO data}

2.3.1 Operational modes. The MEMO experiment mainly deals with the digitized waveform data it produces internally. However, it is also used to transmit: (1) the ELF NVK-ONCH filter-bank data on a regular basis and (2) the VLF NVK-ONCH filter data and the POLRAD sounder data via specific modes.

There are basically three types of MEMO operational modes, namely visualization mode, continuous mode and hybrid mode. The visualization mode, labelled VISU, only produces low-resolution data. One electric field component and one magnetic field component are connected. In order to combine measurements over several frequency bands, sets of ELF or VLF frequency samples are inserted between sets of LF samples. As an example, for each electromagnetic field component, one obtains a set of LF data each $0.2 \mathrm{~s}$, but at the end of each $1 \mathrm{~s}$ interval one receives a set of VLF or ELF data instead of a set of LF data. As a result, when making spectral analyses on the ground, spectrograms with time resolutions of $0.2 \mathrm{~s}$ in LF, and $1 \mathrm{~s}$ in VLF and ELF are obtained.

Continuous mode provides both high-resolution and low-resolution data. The former are obtained from continuous recording, over the longest possible time interval, of waveforms associated with the measurement of 2 to 5 electromagnetic components. DMA data collection is started, either just after the switching-on of the mode, or from a flag. Then, real-time comparison tests are made on the recorded and the measured signal power. When more powerful signals are detected, the DMA data are erased and new data are recorded. At the end of the mode, and if the signal power is large enough the data contained in the DMA memory are transmitted to the ground. During the whole mode duration, lowresolution data, collected as in the visualization mode, are transmitted by the telemetry.

The hybrid mode is basically similar to the continuous mode providing high- and low-resolution data. However, instead of being filled in a single run, i.e. continuously, after on-board tests on the signal power, the DMA memory is filled in 8 to 11 periodic runs, without any tests on the signals. The time periodicity, fixed by telecommands (TC), varies from 8 to $128 \mathrm{~s}$.

2.3.2 Quick look data. Quick looks are constructed from spectrograms of VISU data. There are two views: one for the magnetic and another for the electric field. The total duration is $1 \mathrm{~h}$ in increments of $2.5 \mathrm{~min}$. The view for the magnetic field contains spectrograms of the magnetic component oriented along the spin axis of the satellite . For the sake of simplicity it is noted $\mathrm{Bx}$, in the three frequency bands. The view for the electric field contains spectrograms of the ELF and VLF electric components oriented along the $y$ axis $\left(E_{y}\right)$ plus spectrograms of the LF component rotated by $45^{\circ}$ from the $y$ axis (i.e. from $E_{1}$ ) in the $\mathrm{YZ}$ plane. For the sake of simplicity, a generic label $\left(E_{y}\right)$ is used for the graphs. Due to the non-deployment of one boom unit (see Hanasz et al., this issue) the interpretation of the $\mathrm{E}_{1} \mathrm{LF}$ measurements in terms of an electric field is not straightforward. As a first approximation, the effective length of the antenna is supposed to be half of the length of the boom unit which has been deployed.

Quick looks of the magnetic field are used to test the nature of the field - electrostatic or electromagnetic. According to the sensitivities of the sensors, the events are better detected from electric measurements. Moreover, strong interference lines limit the use of the magnetic spectrograms above $120 \mathrm{kHz}$. Time variations of the amplitude of the lines show that the pollution is due to on-board systems and/or other scientific instruments embarked on the satellite. But the precise source has not been identified so far.

An example of quick look is given in Fig. 4. The data having been sent to a telemetry system (STO) working at $20 \mathrm{kbits} / \mathrm{s}$, the time resolution is $0.2 \mathrm{~s}$ for the LF spectrogram and $1 \mathrm{~s}$ for the ELF and VLF ones. The frequency resolutions are $2.08 \mathrm{kHz}, 208 \mathrm{~Hz}$ and $10.04 \mathrm{~Hz}$ in the LF, VLF and ELF frequency bands, respectively. Gaps in the spectrograms correspond to time intervals during which the high-resolution data are sent to the telemetry system. The wave phenomena will be discussed in the next section.

In first data analysis, AKR harmonics were detected over a $\sim 6$ min time interval on the electric LF spectrogram. However, after having been alerted by the POLRAD experimenters who did not observe any harmonics, we noticed that a dlog function was improperly applied to the MEMO data. This did not produce appreciable changes in the electric spectrograms except for the case mentioned. The dlog function applied to a waveform containing numerous natural or/ and artificial transients generated a non-symetric signal 


INTERBALL-AURORAL 96304_2_S2_1 MEMO(LPCE/CNES)

Day : $27 / 0 c t / 96$ Orbit : 248

Page : 51
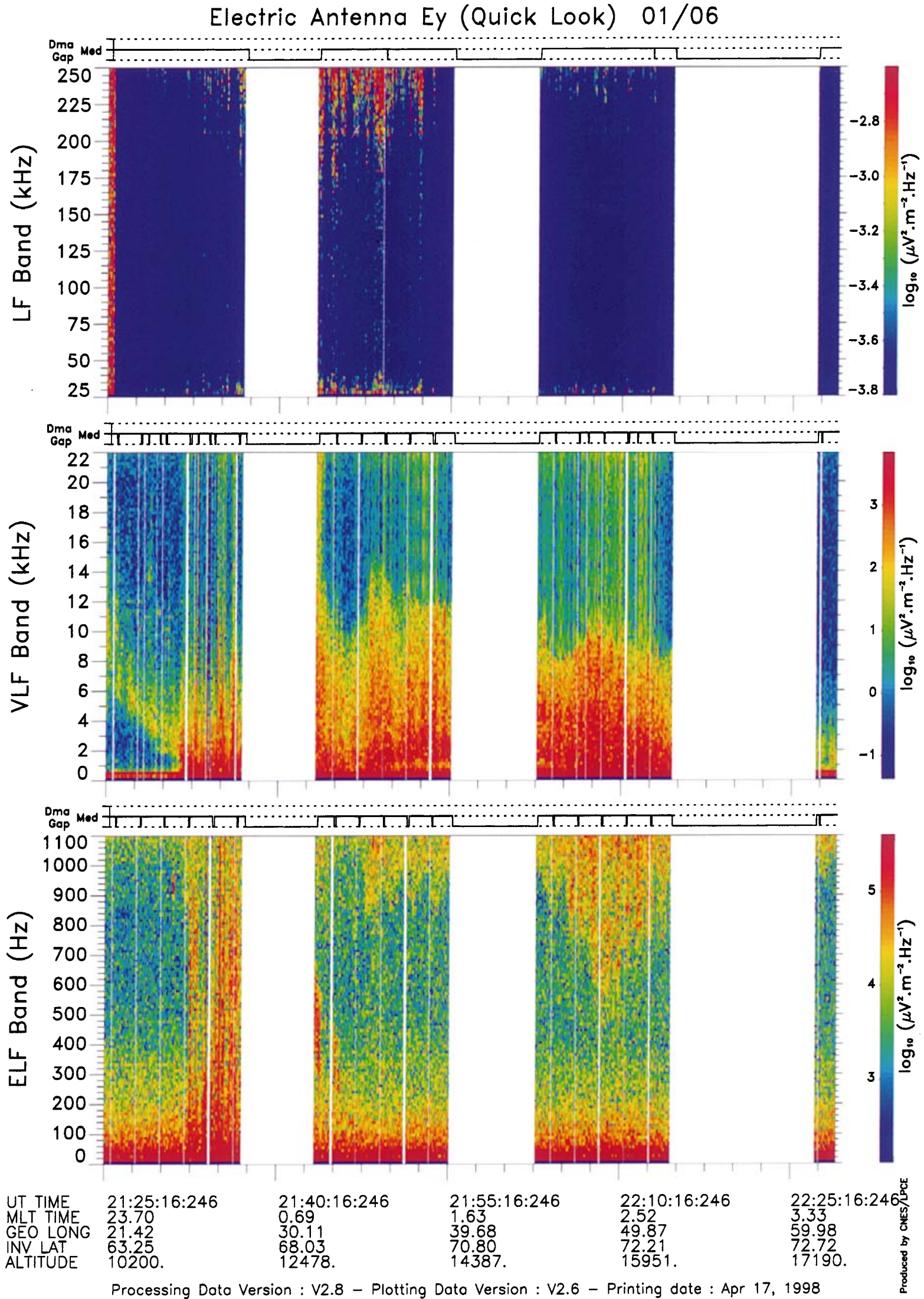
whose the Fourier transform contains harmonics. As a consequence, firstly, we do not claim any more that AKR harmonics are seen on INTERBALL 2, and secondly, we advise the experimenters to avoid any nonlinear transform in their data processing.

2.3.3 First-order propagation characteristics. The waveforms recorded in the DMA memory are mainly used to estimate the propagation characteristics of the observed waves. First order analyses are described in Lefeuvre et al. $(1982,1986)$. Here, magnetic data are considered only. The corresponding software is included in the SWAN library developed at LPCE (Lagoutte and Latrémolière, 1997a,b). The propagation characteristics are derived from the estimated values of the $3 \times 3$ spectral matrix of the 3 magnetic field components. One works in a Cartesian coordinate system OXYZ in which the $\mathrm{OZ}$ axis is parallel to the measured Earth magnetic field direction $\mathbf{B}_{0}$.

Let $\mathbf{S}(f)$ be the corresponding spectral matrix of the magnetic wave-field, field components at the frequency $f$, then it writes:

$$
\mathbf{S}(f)=\begin{array}{rrr}
S_{x x}(f) & S_{x y}(f) & S_{x z}(f) \\
S_{y x}(f) & S_{y y}(f) & S_{y z}(f) \\
S_{x z}(f) & S_{z y}(f) & S_{z z}(f)
\end{array}
$$

with $S_{i j}(f)$ the cross-power spectrum of the components $B_{i}$ and $B_{j}$ (estimated here using the periodogram method, i.e. by taking $S_{i j}(f)=E\left[B_{i}(f) B_{j}{ }^{*}(f)\right]$ with $E[\psi]$ the mathematical expectation of $\psi, B_{i}(f)$ the Fourier transform of $B_{i}(t)$ and $B_{i}(f)^{*}$ its complex conjugate).

A degree of polarization, also named percentage of polarization (Samson, 1973 Samson and Olson, 1980; Pinçon et al., 1992), is estimated from the relation

$$
P(f)=\left\{3 \operatorname{Tr}\left[\mathbf{S}^{2}(f)\right]-(\operatorname{Tr}[\mathbf{S}(f)])^{2}\right\} /\left\{2\left(\operatorname{Tr}[\mathbf{S}(f)]^{2}\right\}\right.
$$

It takes values between $[0,1]$. Values close to 1 (generally between 0.9 and 1.) mean that the observed wave may be considered as a plane wave. Lower values mean that the plane wave approximation is not valid and that other methods must be used to determine the propagation characteristics (Storey and Lefeuvre, 1979, 1980). Values exactly equal to 1 are suspicious. They generally correspond to monochromatic waves of artificial origin, i.e. to interferences. Linear polarized waves tend to have values close to 1 .

Assuming the wave is plane, one can determine the ellipticity $E$ of the polarization ellipse. Here, following Samson and Olson (1980), we estimate it from the eigenvector $\mathbf{V}$ associated with the non-zero eigenvalue of the spectral matrix $\mathbf{S}(f)$. If we note $\mathbf{V}=\mathbf{R}_{1}+j \mathbf{R}_{2}$, we have:

Fig. 4. A nightside pass through the auroral zone and the polar cap, on 27 October, 1996, showing wave emissions in the three frequency bands. The figure is a copy of the electric view of the MEMO Quick Look data (see text). The time interval between two time marks is $2 \min 30 \mathrm{~s}$
$E=\left\|\mathbf{R}_{2}\right\| /\left\|\mathbf{R}_{1}\right\|$

In the plane wave hypothesis, one may determine the wave normal direction $\mathbf{k}$ from the spectral matrix $\mathbf{S}(f)$. It is characterized by the $\theta$ and $\phi$ angles. The angle is the angle made by the $\mathbf{k}$ vector with $\mathbf{B}_{0}$. The angle is the angle between the projection of the $\mathbf{k}$ vector onto the OXY plane and the OX axis. When using the magnetic components only, one cannot make the distinction between a $\mathbf{k}$ vector directed in the $\mathbf{B}_{0}$ direction $\left(\theta=\theta_{0}\right.$, with $\theta_{0}<90^{\circ}$, and $\left.\phi=\phi_{0}\right)$ and in the opposite direction $\left(\theta=180^{\circ}-\theta_{0}, \phi=\phi_{0}+180^{\circ}\right)$.

The estimators we used here for the $\theta$ and $\phi$ angles have been defined by Means (1972). They are based on the imaginary part of the spectral matrix. They are

$$
\begin{aligned}
\operatorname{Cos} \theta= & \operatorname{Im}\left[S_{x y}(f)\right] /\left\{\operatorname{Im}^{2}\left[S_{x y}(f)\right]\right. \\
& \left.+\operatorname{Im}^{2}\left[S_{x z}(f)\right]+\operatorname{Im}^{2}\left[S_{y z}(f)\right]\right\}^{1 / 2} \\
\operatorname{tg} \phi=- & \operatorname{Im}\left[S_{x y}(f)\right] / \operatorname{Im}\left[S_{x z}(f)\right]
\end{aligned}
$$

In theory the modulus of $\cos \theta$ is physically meaningfull only. In practice, one can demonstrate (Lefeuvre et al., 1986; Parrot et al., 1989) that its sign gives a good indication about the sense of polarization, even when the plane wave approximation is not valid. It is positive for a right-hand polarization $(R)$ and negative for a lefthand $(L)$. Then, here after, the following associations will be made:

$$
\begin{aligned}
& \theta<90^{\circ} \leftrightarrow \mathrm{R} \text { waves } \\
& \theta>90^{\circ} \leftrightarrow \mathrm{L} \text { waves }
\end{aligned}
$$

Obviously, values around $90^{\circ}$ are the most sensitive to the background noise. One must be very careful when interpreting them.

At the present stage of the analysis, where electric data are not included and where attitude data are missing, there is no direct possibility firstly, to remove the ambiguity on the $\theta$ and $\phi$ values, and secondly, to fix the $\phi$ angle as regards the geomagnetic meridian plane.

\section{AKR emissions and associated phenomena}

Two orbits have been chosen to illustrate the MEMO observations of AKR emissions and associated phenomena. Both correspond to crossings of the radiation cone of an AKR source just above the source.

\subsection{The 27 October, 1996, orbit}

Let us return to Fig. 4. Examining the orbit parameters one notes that, after having crossed the auroral zone, at magnetic local time (MLT) values between 23.70 to $\sim 01.00 \mathrm{H}$ and altitude values between 10200 and 13 $000 \mathrm{~km}$, the satellite stays approximately on the same $L$ shell (Invariant latitude around $72^{\circ}$ ) at the inner boundary of the polar cap. During the later period the MLT values run from 01.00 to $03.30 \mathrm{H}$ and the altitude from 13 000 to $17000 \mathrm{~km}$. Several wave phenomena are observed in the three frequency domains. In order to develop some 
idea about the variations of the local plasma frequency $f_{\text {pe, }}$ let us analyze the auroral hiss emission observed from 21:32 to 22:15 UT in the VLF band.

Propagation characteristics may be estimated each 32 s, i.e. when waveforms are recorded in the DMA memory. The results obtained around 22:07:08 UT are given in Fig. 5. The top panel represents a $\sim 0.1 \mathrm{~s}$ averaged power spectrum of the Bx component. It has a main peak between 0.70 and $1.75 \mathrm{kHz}$ and secondary peaks with approximately same amplitudes between 1.75 and $4.1 \mathrm{kHz}$. The high cutoff frequency is not very well defined. The power spectrum above $\sim 9 \mathrm{kHz}$ is associated with the background noise. Applying the polarization test (5) to the fifth panel (Theta angle) we note that except perhaps at $1.1 \mathrm{kHz}$ where the test is not valid $\left(\theta \sim 90^{\circ}\right)$, the waves below $1.75 \mathrm{kHz}$ are left
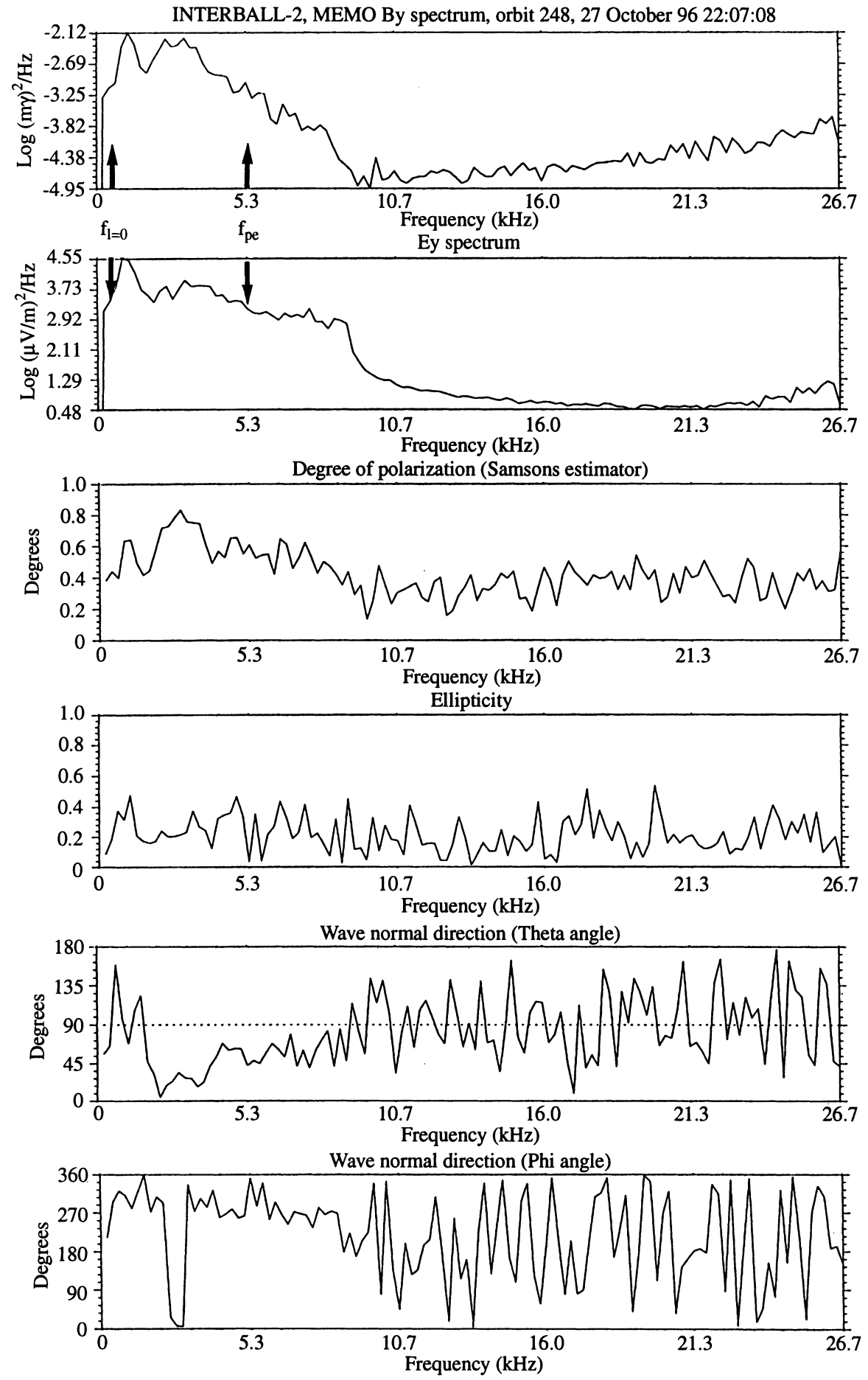

Fig. 5. Wave analysis over a $0.1 \mathrm{~s}$ time interval of the auroral hiss of Fig. 4. The time interval is centered around 22:07:08 UT. From top to bottom are represented: the auto-power spectrum of the $\mathrm{Bx}$ component, the auto-power spectrum of the Ey component, then the propagation parameters derived from sub-section 2.3.3: degree of polarization, $\theta$ and $\phi$ angles 
polarized whereas the waves above $1.75 \mathrm{kHz}$ are right polarized. The second panel represents the power spectrum of the Ey electric field component. The peak below $1.75 \mathrm{kHz}$ is much higher than all other peaks. There is a well defined high cutoff frequency around $9.5 \mathrm{kHz}$. We will examine in the next paragraph how it can be interpreted. The third panel provides an estimate of the degree of polarisation. Although all values are rather low, one observes that the degree of polarization is higher below $9.5 \mathrm{kHz}$, where a wave is detected, than above, where a noise is measured. The same observation applies for the two last panels. Although the plane wave approximation is not valid, the $\theta$ and $\phi$ values obtained below $9.5 \mathrm{kHz}$ show some consistency. Being, in some sense, average values of full wave distribution functions (see Lefeuvre et al., 1982; Lefeuvre and Helliwell, 1985) they show a regular variation in frequency of all wavenormal directions $\mathbf{k}$. As far as the ellipticity parameter is concerned (fourth panel) there is no clear interpretation.

Returning to the two upper panels, it is clear that if the frequency peak below $1.75 \mathrm{kHz}$ corresponds to an $L$ mode, the low cutoff frequency at $0.75 \mathrm{kHz}$ is the $L=0$ cutoff $\left(f_{L=0}\right)$. Its analytic expression is given by $f_{L=0}=-f_{c e} / 2+\left(\left(f_{c e} / 2\right)^{2}+f_{p e}\right)^{1 / 2}$, with $f_{c e}$ the local electron gyrofrequency measured on-board (here $36.6 \mathrm{kHz}$ ). From the $f_{L=0}$ value one may derive the $f_{p e}$ value, which is $5.3 \mathrm{kHz}$. This value is indicated by an arrow on the Ey power spectrum in Fig. 5. It corresponds to a modification in the slope of the spectrum. This can be interpreted as a boundary between a propagation in the whistler mode (below $5.3 \mathrm{kHz}$ ) and in the $\mathrm{Z}$ mode (above $5.3 \mathrm{kHz}$ ). Similar transitions have already been pointed out on DE 1 data by Calvert and Hashimoto (1990). In such a case the dramatic decrease seen at $8.9 \mathrm{kHz}$ would be the $\mathrm{Z}$ mode high cutoff frequency. It is much below the upper hybrid resonance $\mathrm{f}_{U H R}=\left(f_{p e}^{2}+f_{c e}^{2}\right)^{1 / 2}$, which is the theoretical upper boundary for perpendicular propagation, indicating that the observed $\mathrm{Z}$-mode waves are propagating nearly parallel to B. Now, Bahnsen et al. (1989) have already indicated the difficulty of measuring the electronic density in low-density conditions. One important consequence is that, in the absence of a clearly identified $L=0$ cutoff we are in the same situation as for DE 1 to determine $f_{p e}$. One must look either from a natural resonance of the medium or for what seems to be the transition between the whistler mode and the right polarized $\mathrm{Z}$ mode. In the present case, where the $\mathrm{Z}$ mode is too electrostatic to be seen on the magnetic data (not shown), one can correct $f_{p e}$ from the Bx power spectrum. But, there is no guarantee that this is always the case. Note that the low amplitude of the magnetic field components above $5.3 \mathrm{kHz}$, and the probable mixing of the whistler and $\mathrm{Z}$ modes below that frequency, could explain the very low values obtained for the degree of polarisation.

Unfortunately, we do not have high-resolution data on a continuous basis to detect the $L$ mode, then to locate the $L=0$ cutoff, in order to follow the variations of the $f_{p e}$ values along the orbit. So, we are obliged to look for slope changes in the power spectra, and/or to compare low and high cutoff frequencies in order to check if the observations are consistent with a low or a high plasma frequency value (as an example, $f_{L=0}=0$ implies $f_{p e}=0$ ). Applying these rules to the full sequence presented in Fig. 4, one concludes that regions of low $f_{p e}$ values, i.e. of possible auroral density cavity (Calvert, 1981), seem to be crossed around 21:34, 21:47, 21:51 and 22:05 UT.

During the whole of this time interval, the POLRAD experiment (see Hanasz et al., this issue), not shown here, detects AKR emissions. The frequency band extends from $\sim 100 \mathrm{kHz}$ to $\sim 600 \mathrm{kHz}$. V-shaped structures are approximately centered around 21:36, 21:39, 21:43 and 21:48 UT. The bottom frequencies are detected by MEMO (see Fig. 4). However, due to a data gap, MEMO misses the strongest event at $\sim 21: 39$ UT where the minimum frequency $(\sim 100 \mathrm{kHz})$ is the closest to the local electron gyrofrequency $(\sim 60 \mathrm{kHz})$, i.e. where the distance between the source and the point of observation is minimum. For the other events, a modulation at half the spin frequency does not allow us to identify clearly the heads of the $\mathrm{V}$ structures and finer temporal structures superposed on them. It is clear that the minimum frequencies tend to increase when one moves away from the strongest event. The power collected on the magnetic antennas is too weak to estimate the propagation characteristics.

Measurements performed by the ION experiment (Sauvaud et al., this issue) in the same time interval show: (1) a flux of low-energy electrons $(<30 \mathrm{ev})$ from 21:28:40 to 21:31:25 UT, with part being upgoing, which is in agreement with the observation of the left branch of a saucer before 21:29 UT; (2) an intense inverted V precipitation (21:31:25 and 21:41:40 UT), with energies reaching $10 \mathrm{kev}$ at 21:36:15 UT, and (3) a counting rate too low to be interpreted.

By assembling all these observations we come to with the following scenario. In the auroral part of its orbit (i.e. before $\sim 21: 40$ UT) INTERBALL 2 crosses the outer part of an acceleration region. At about the same time it encounters the emission cone corresponding to high frequencies generated at lower altitudes (see i.e., the $\mathrm{s} / \mathrm{c}$ position in Fig. 2 of Benson and Calvert, 1979). The data gap in MEMO does not allow us to define the cone better and to evaluate the variations of the local electronic density. Once in the polar cap region, the satellite stays close enough to cross other cones of AKR and to detect traces of density cavities. Referring to the number of $\mathrm{V}$-shaped structures one concludes that over a $40 \mathrm{~min}$ interval, or, if we prefer over a $25^{\circ}$ longitude span the satellite sees four to five independent AKR point sources.

An ELF/VLF turbulence phenomenon observed from 21:32 to 21:37 UT seems to be part of the process that takes place in the acceleration region. However, a pulse detected just before ( 21:31 UT) around $1.7 \mathrm{kHz}$ by NVK-ONCH (Mogilevsky et al., this issue) could be related to a Tromsö heating experiment. 

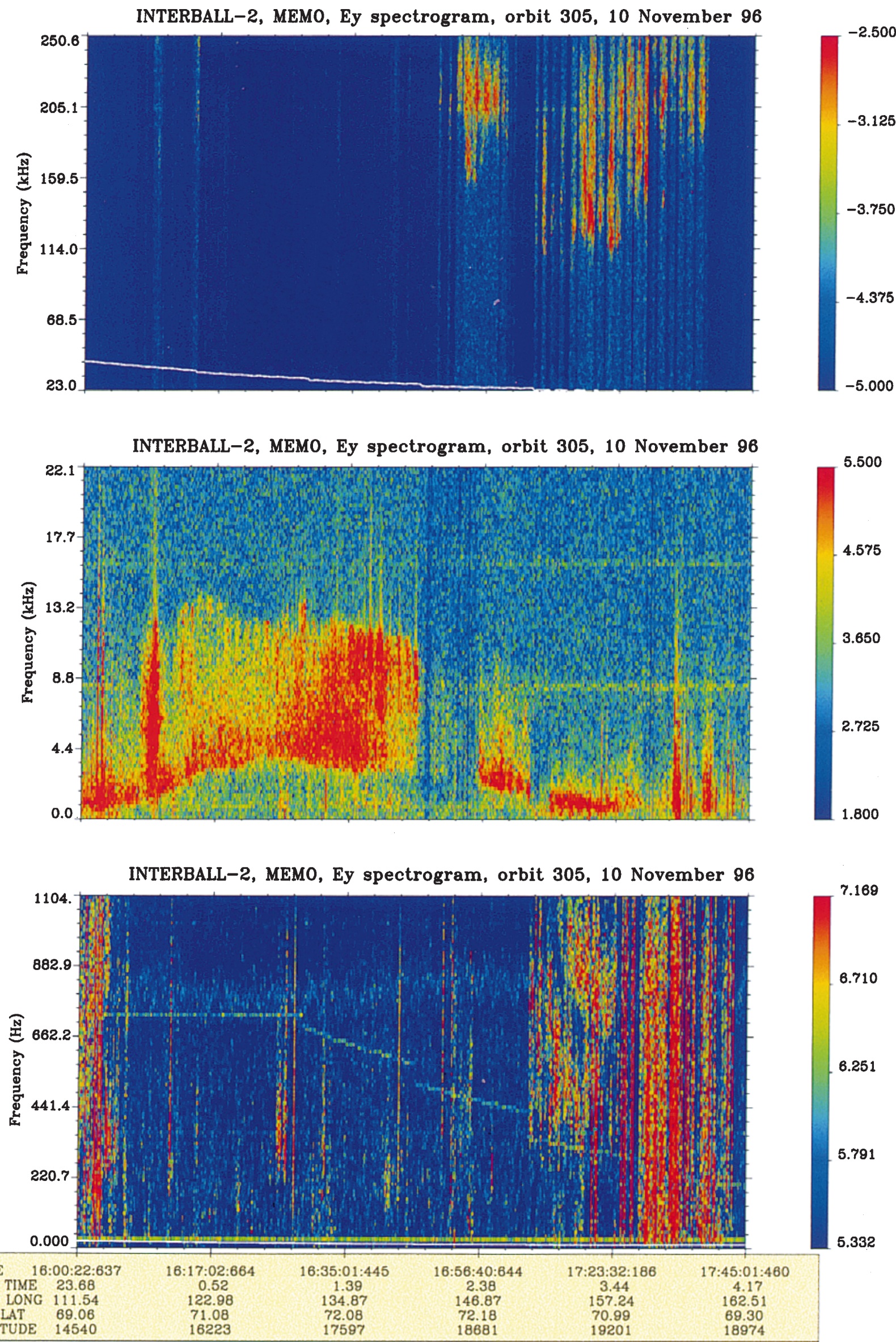


\subsection{The 10 November, 1996, orbit}

Figure 6 presents a more detailed example of the crossing of the radiation cone of an AKR emission. The format is not the same as Fig. 4. The graphs have been reconstructed by adding spectrograms obtained over non-contiguous time intervals. The gaps associated with the transmission of high-resolution data to the telemetry system have been ignored. As a consequence, whereas the UT times indicated in Fig. 6 are correct, the distances between the time marks may vary. Due to changes in the signal to noise ratio over a few time intervals, gain adjustments introduce spurious noises in the spectrograms. They are ignored here. This explains apparent decreases in the VLF signal around 16:50 UT and 17:00 UT. Comparisons between electric and magnetic measurements show that the VLF and ELF emissions are practically electrostatic. The local gyrofrequency value $\left(f_{c e}\right)$, estimated from the IMAP3 data (Arshenkov et al., this issue) is indicated by a white line on the LF spectrogram. It is always well below the AKR lowest frequency. An examination of the orbit parameters shows that the satellite is at its apogee around 17:23 UT, and that, during one full hour (16:45-17:45 UT), altitude variations $(600 \mathrm{~km})$ and invariant latitude variations $\left(3^{\circ}\right)$ are very small. In a first approximation, one can consider that the satellite stays on the same $L$ shell and explores the AKR emission as a function of longitude.

In some respects, the VLF radiation spectrum is in between the funnel-shaped spectrograms of auroral hiss observed on DE 1 (Gurnett et al., 1983) and the Vshaped spectrograms observed on AKEBONO (Kasahara et al., 1995). The right branch of a "V" structure may be drawn from $\sim 16: 00$ to $16: 23$ UT and a left branch from $\sim 16: 23$ to $17: 30$ UT. One difficult point is to know whether the lowest extremity of each branch is associated with a density cavity or not. According to the electrostatic character of the emissions, a full determination of the propagation characteristics is very difficult. However, whatever the propagation mode in the VLF hiss band, it is clear that the $f_{p e}$ values are at the minimum when the upper and lower cutoff frequencies have both very low values. As a consequence one can consider that the satellite crosses two density cavities: one around 16:00 UT and the other around 17:30 UT. Measurements performed by the ION experiment detect: first, an inverted $\mathrm{V}$ distribution of $<10 \mathrm{kev}$ electrons (15:40-16:05 UT), then an intense flux of precipitating electrons below 1 kev (17:35-17:55 UT). Moreover, they show upgoing protons of $\sim 1 \mathrm{kev}$ around 17:30 UT. At that time, lower energy upgoing protons are also detected on the HYPERBOLOID experiment (Dubouloz et al., this issue). Under these conditions, the

Fig. 6. A nightside pass over a source of AKR emissions on 10 November 1996. The figure has been constructed from several spectrograms, similar to those of Fig. 4, without taking into account the time gaps. The UT times which are indicated are exact but the time interval between two time marks may vary. The units are arbitrary (see text)
Gurnett et al. (1983) model applies. The radiation pattern is consistent with an emission produced below the satellite and propagating with a wave normal angle within or close to the resonance cone. The emission generation mechanism may be associated with the Landau resonance.

Now, let us examine the wave population within the two density cavities. For the first one, the satellite is probably too far from the cavity center to cross any AKR emission below $250 \mathrm{kHz}$. However, an ELF turbulence is detected close to 16:00 UT and a broadband electrostatic noise (BEN, described e.g., by Potelette et al., 1987) seems to develop around 16:10 UT. The observations made within the second cavity are much more informative. An AKR emission is clearly centered around the minimum $f_{p e}$ value. Its minimum frequency $(\sim 110 \mathrm{kHz})$ is well above the upper hybrid frequency $f_{U H R}=\left(f_{c e}^{2}+f_{p e}^{2}\right)^{1 / 2}$. At the same time an ELF emission is seen. Curiously it has two frequency bands, one approximately between 400 and $700 \mathrm{~Hz}$, the other above $700 \mathrm{~Hz}$. At this stage (where the transfer functions of the filters have not been corrected) it is difficult to know whether the upper band is the low frequency part of the VLF auroral hiss emission or not. In any way, none of the two bands is associated with the local proton gyrofrequency (here $\sim 15 \mathrm{~Hz}$ ). As in the first case, an ELF turbulence and BEN emissions are seen on the right part of the cavity. Investigations have to be made in collaboration with the IESP experimenters (Perraut et al., this issue) which make measurements between 0.1 and $30 \mathrm{~Hz}$.

A detailed analysis of the propagation characteristics of the AKR event has been performed over a $10 \mathrm{~ms}$ time interval. The results are given in Fig. 7. Except perhaps at $132 \mathrm{kHz}$ and $263 \mathrm{kHz}$, where the degree of polarization is not exactly equal to one (see the interpretation of the $P(f)$ values in 2.3.3), all the frequency peaks observed in the top panel $(44,65,98,132,163,197$, $205,244 \mathrm{kHz})$ are due to interferences on the LF magnetic antennas. However, although being corrupted by a power leakage from one Fourier component to the other, the propagation parameters derived from the spectral matix of the magnetic field components, out of the frequency peaks, are consistent enough to be considered as physically meaningful. Due to calibrations problems, the units taken for the LF electric components are indicative only.

Finally, the following points are clear:

1. A first band of AKR is detected between 120 and $170 \mathrm{kHz}$. It corresponds to the bottom part of the AKR event of Fig. 6. According to the values of the degree of polarization $(\sim 0.8)$ out of the interferences, the plane wave approximation is not valid. Then, the estimated propagation parameters are indicative only. If, the ellipticity value is effectively of the order of 0.8 , the polarization is quasi-circular. This is consistent with the apparent isotropic distributions in the electric and magnetic power densities. The $\theta$ values being always much lower than $90^{\circ}$, the waves are right polarized whatever the dispersion in $\mathbf{k}$ vectors. The propagation mode is clearly the $R$-X mode. As regards to the values, 

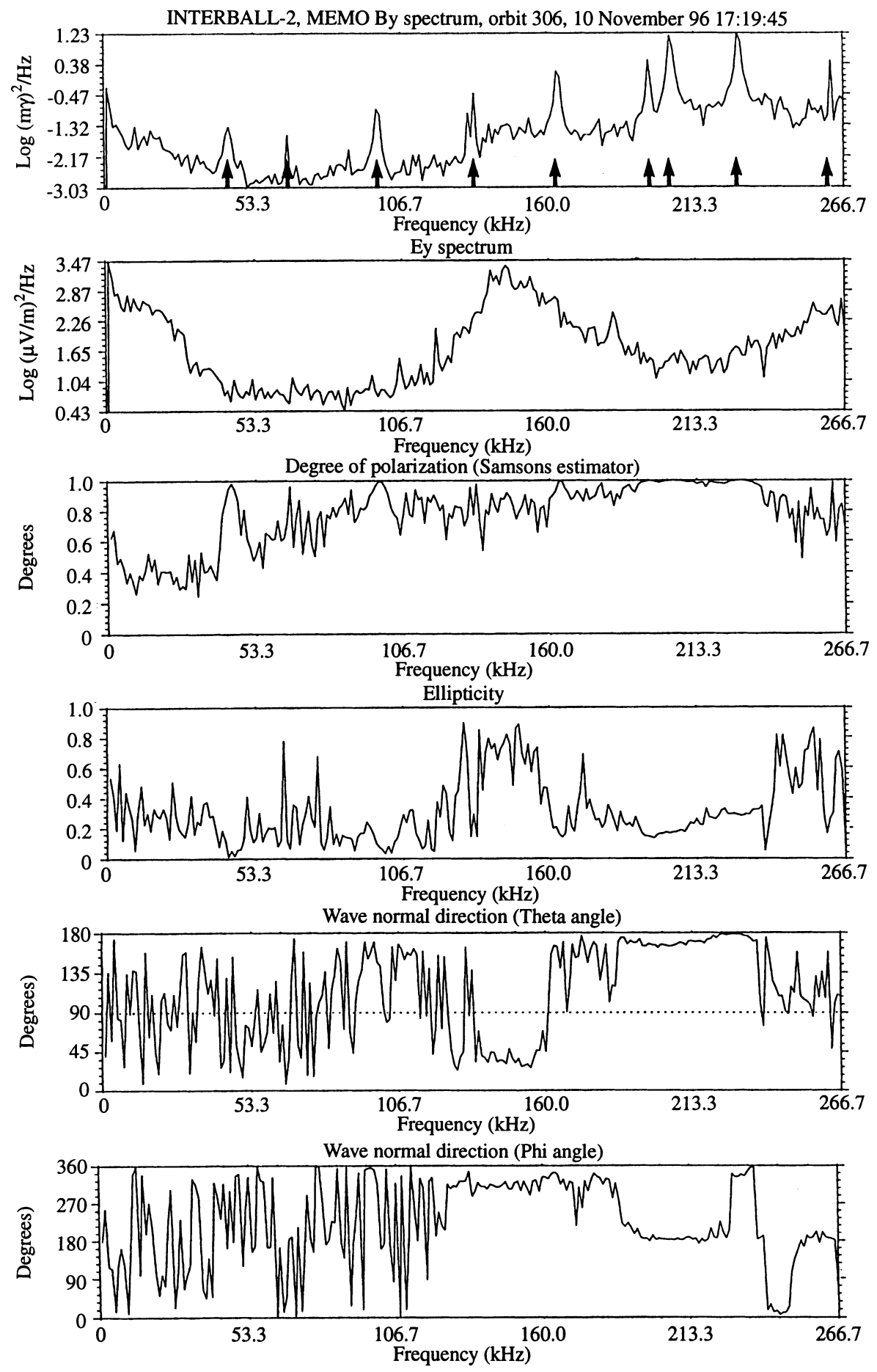

Fig. 7. Wave analysis over a $10 \mathrm{~ms}$ time interval of the AKR emission of Fig. 6. The time interval is centered around 17:19:45 UT. Interference frequencies are indicated by arrows on the top panel

they are nearly constant out of the interferences. Assuming the wave distribution function is one-peaked (which has to be checked from a wave distribution function analysis), one may consider that the $\mathbf{k}$ vectors are contained in a narrow cone centered around a $\mathbf{k}_{0}$ vector making a weak angle $\left(\sim 30^{\circ}\right)$ with the Earth's magnetic field direction. As the source is below the satellite, the propagation cone is probably oriented upwards. The $\phi$ angle has a constant value equal to $\sim 320^{\circ}$, but, without attitude data, we cannot interpret it in terms of a propagation in or off the geomagnetic meridian plane.

2. A second band of AKR is detected between 170 and $240 \mathrm{kHz}$. It is not seen on Ey at the UT time of the analysis, but is clearly observed before and after on the

Fig. 8. Examples of fine structures in AKRs 
INTERBALL-2, MEMO, Ey spectrogram, orbit 256, 29 October 96
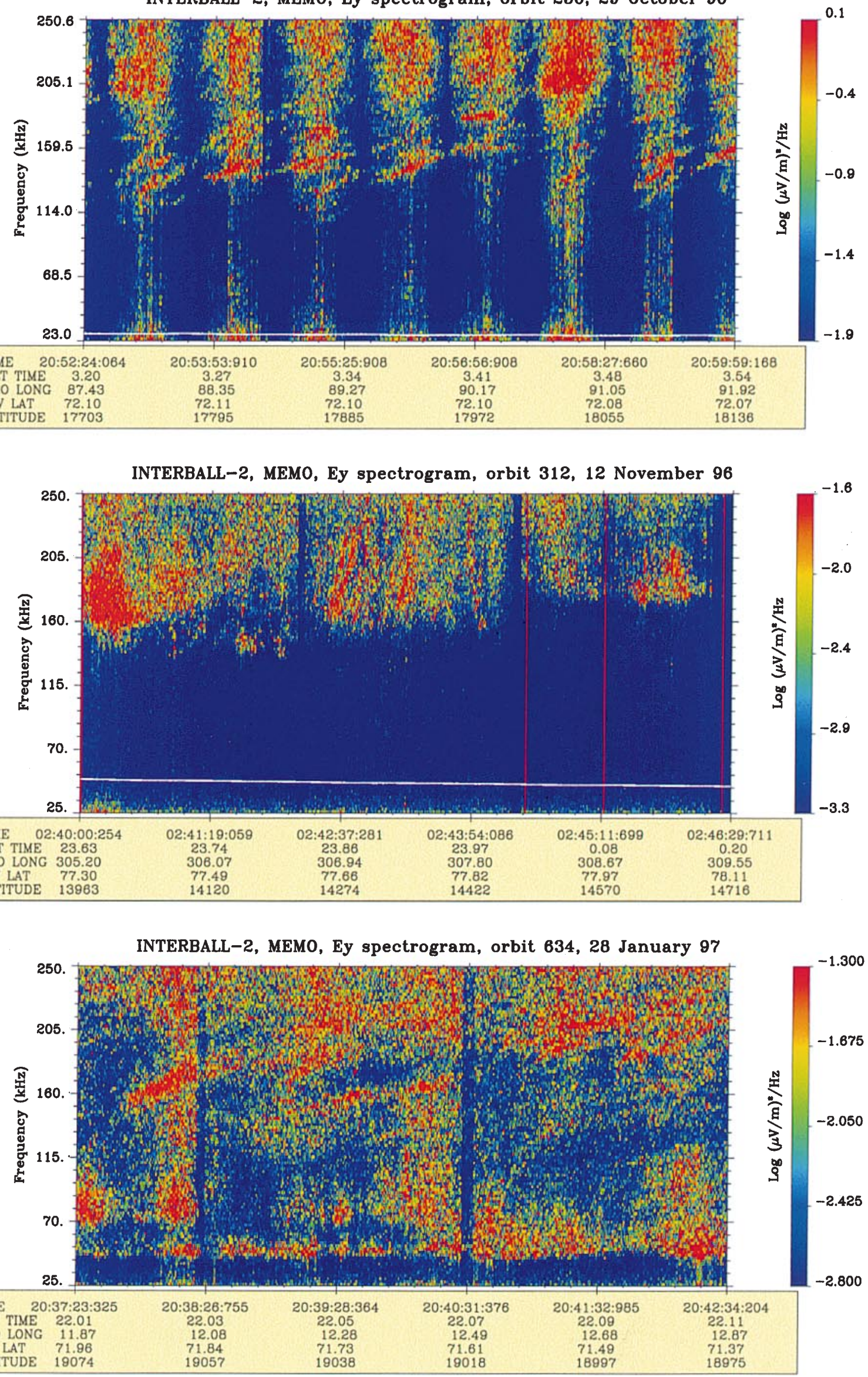
spectrogram of Fig. 6. The high values of the degree of polarization means that the plane wave hypothesis is valid. The low value of the ellipticity (slightly below 0.3 ) shows that the polarization tends to be linear. This is consistent with strong variations of the field amplitude during a spin period. In the whole frequency band the emission is left polarized $\left(\theta>90^{\circ}\right)$, i.e. propagates in the $L$-O mode. The $\theta$ angle has a value equal to $\sim 15^{\circ}$, which means that the propagation is quasi-parallel. Again, in the absence of attitude data, one cannot fully interpret the constant value $\left(\sim 160^{\circ}\right)$ of the $\phi$ angle. However, if the propagation is upwards in the two AKR frequency bands, one observes that the azimuthal directions of the $R$-X and $L$-O modes are nearly opposite.

The observation of AKR emissions at $f \gg f_{c e} \gg f_{p e}$, in a region of downward-directed electron beams and upward ions suggests that the waves propagate upwards from the source to the point of observation following approximately the same line of force as the accelerated particles. A ducting process seems to take place. Such an interpretation is supported by the low values obtained for the $\theta$ angle at the center of the $\mathrm{V}$-shaped structure of Fig. 6. In another way, a confinement of the waves in the source region and just above is present in several generation mechanisms of AKR. It is implicit in the feedback model of Calvert $(1981,1982)$ where a wave amplification is produced by a reflexion of well-defined $\mathbf{k}$ vectors within the auroral cavities. It is explicit in the filamentary source model of Louarn and Le Quéau (1996a, b) where the altitude at which the divergence occurs is evaluated (Louarn, 1997). By analogy with the terminology used in the diffraction theory (see e.g., Born and Wolf, 1980), during the pass of Fig. 6, the satellite seems to cross the Fresnel region for the lowest frequencies (near zone) and the Frauhhoffer region for the highest (far zone). Among the points to solve one has to determine whether the observed propagation modes ( $R$-X at the lowest frequencies and $L-\mathrm{O}$ at the highest) are those produced within the source or are the results of mode couplings in the course of the propagation.

\section{AKR fine structures}

\subsection{MEMO observations}

Although discrete structures have often been pointed out on high-resolution spectrograms, coherent fine structures are observed from time to time only. Figure 8 presents three different cases. They all correspond to night time passes. In each case the satellite follows approximately the same line of force during the whole time of observation. When above $23 \mathrm{kHz}$, the local electron gyrofrequency is represented by a white line.

Examples of fine narrow-bandwidth drifting structures, similar to those reported by Gurnett et al. (1979), Gurnett and Anderson (1981), and Morioka et al. (1981) etc., are shown in the top and bottom panels. The amplitude modulations seen in the top panel is due to the spin rotation (with half period of $\sim 1 \mathrm{~min}$ ). The corresponding waves are probably well polarized i.e., have a very weak dispersion in wave normal directions. The drift rates of the bursts have $\mathrm{d} f / \mathrm{d} t$ values of the order of 0.4 to $0.8 \mathrm{kHz} \mathrm{s}^{-1}$. Following Gurnett et al. (1979) they may be interpreted as a movement of an AKR source region along a magnetic field line. Assuming a radial gradient in the electron gyrofrequency and plasma frequency of the order of $\mathrm{d} f / \mathrm{d} R=0.2 \mathrm{kHz} / \mathrm{km}$, one obtains as a value of the velocity of the source $v_{s}=$ $\mathrm{d} R / \mathrm{d} t=(\mathrm{d} R / \mathrm{d} f)(\mathrm{d} f / \mathrm{d} t)=2$ to $4 \mathrm{~km} / \mathrm{s}$, which is slightly less than the estimates generally published. Since the drift rate is positive and the plasma parameters are decreasing when the altitude is increased, the source motion is supposed to be oriented towards the Earth. These results are consistent with an emission generated just above the local $f_{c e}$ value and moving at a velocity close to the ion acoustic speed.

Now, if the Gurnett et al. (1979) model is correct, taking into account the fact that INTERBALL 2 stays along the same line of force during long time intervals, one can be more precise about the source regions. For the events observed in the top panel of Fig. 8, the spin modulation plus the apparent periodicity in the drifting structures, could indicate: firstly, that the source is located on another line of force (probably at lower invariant latitude) and secondly, that it consists of multiple point sources having a very weak extension in longitude and/or in MLT values. As far as the bottom panel is concerned one has probably to consider two types of sources. One, moving towards the Earth with the velocity of the satellite, to explain the stationary structures observed below $110 \mathrm{kHz}$. Another, moving towards the Earth at a velocity exceeding that of the satellite, to explain the drifting structures observed above $120 \mathrm{kHz}$

The same model can be used to interpret the structures superimposed on a broad-band AKR spectrum in the middle panel of Fig. 8. As an example, for the chorus-like events seen around 02:42:37 UT, the velocity could reach $80 \mathrm{~km} \mathrm{~s}^{-1}$.

Now, the observations on the top panel of Fig. 8 of spectral components occurring as multiplets with a spacing of roughly $20 \mathrm{kHz}$ implies the existence of a non-linear process taking place in the source region. One obvious candidate for this process is the Calvert (1982) feedback source model in which, partial wave reflections at the inner boundaries of the density cavities allow amplified waves to be routed back to their origin. This creates a closed-loop oscillator system which generates waves at discrete frequencies. However, as in Calvert (1982), the observed frequency banwiths we observe $(\sim 1 \mathrm{kHz})$ are much greater than the predicted ones $(\sim 10 \mathrm{~Hz})$. Other models such as the filamentary source model of Louarn and Le Quéau $(1996 a, b)$ must be examined.

Fig. 9. Propagation characteristics of an AKR element of the bottom panel of Fig. 8. A $0.32 \mathrm{~s}$ time interval is analyzed. It starts at 20:37:23:325 UT, i.e., just at the beginning of the recording. 


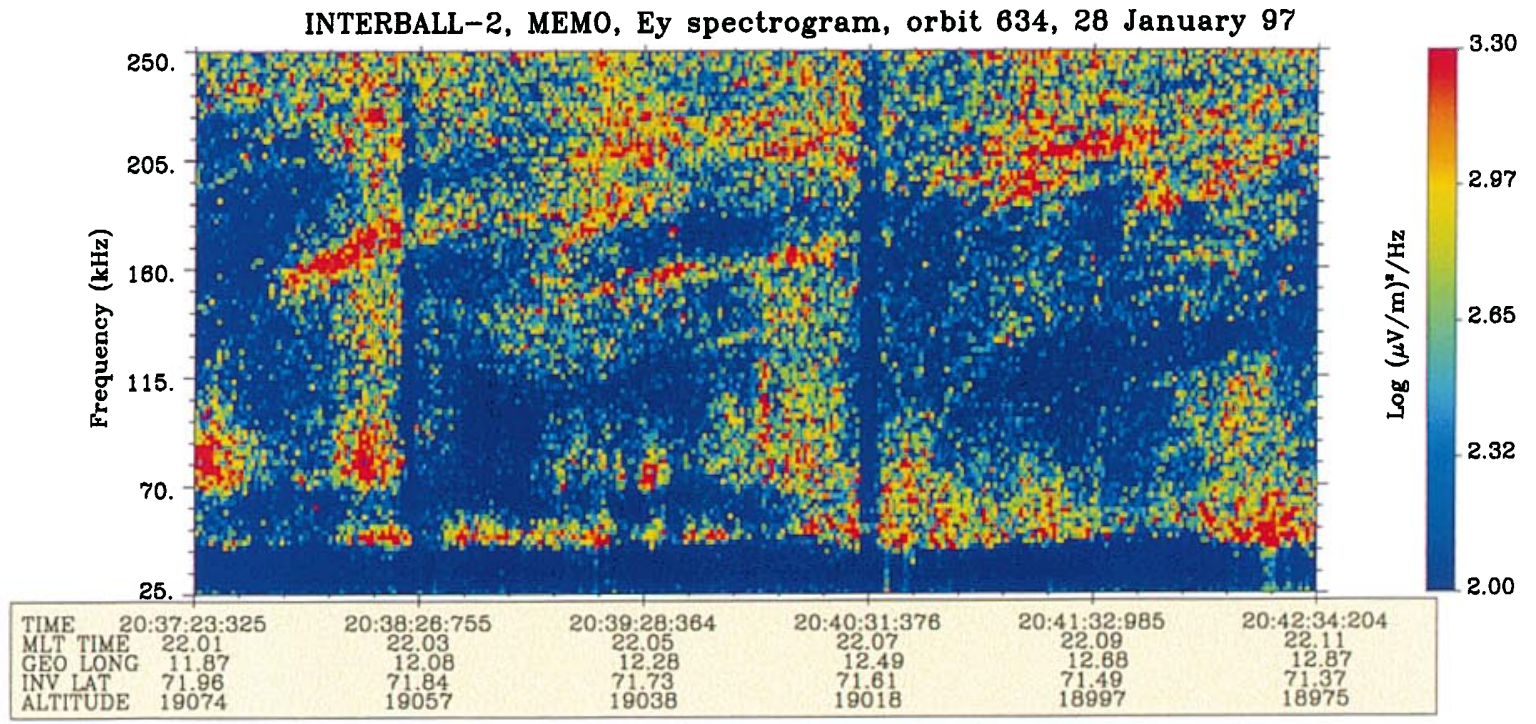

INTERBALL-2, MEMO, Bx spectrogram, orbit 634, 28 January 97

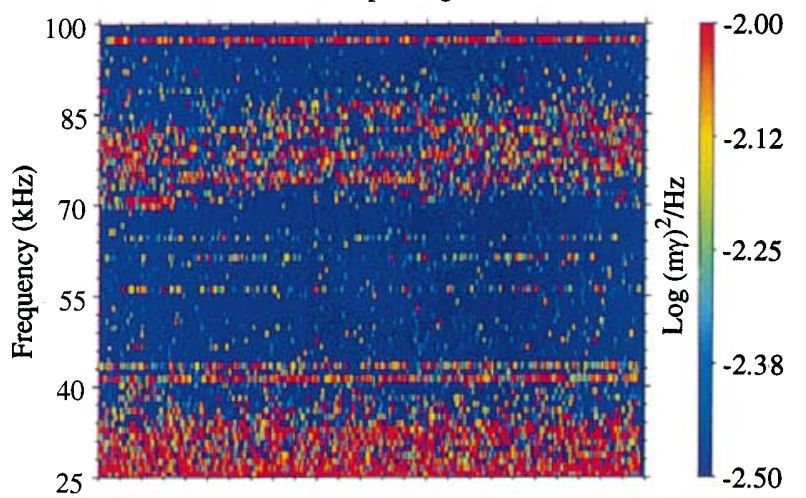

INTERBALL-2, MEMO, Ey spectrogram, orbit 634, 28 January 97
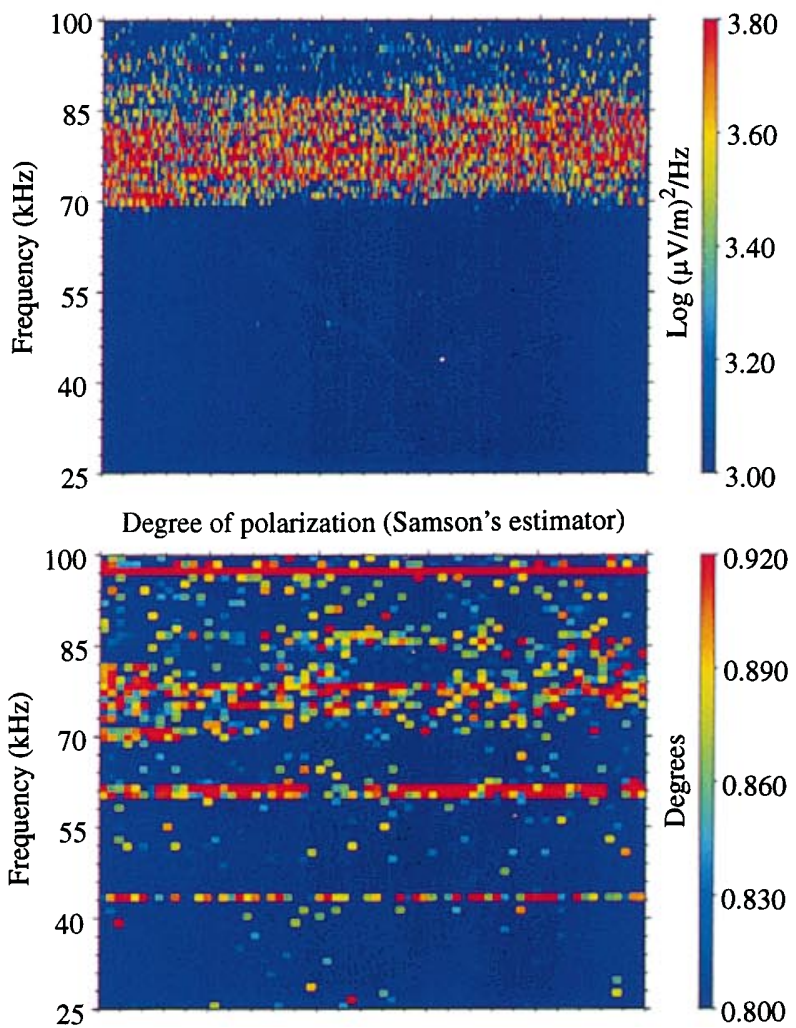

Ellipticity

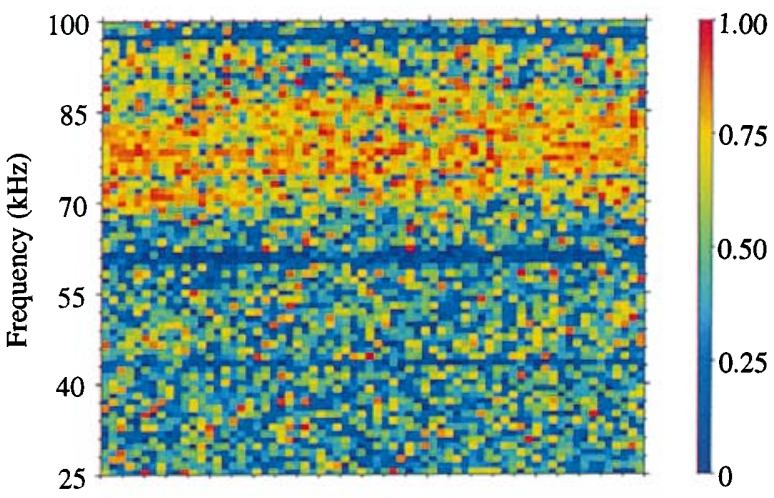

Wave normal direction (Theta angle)
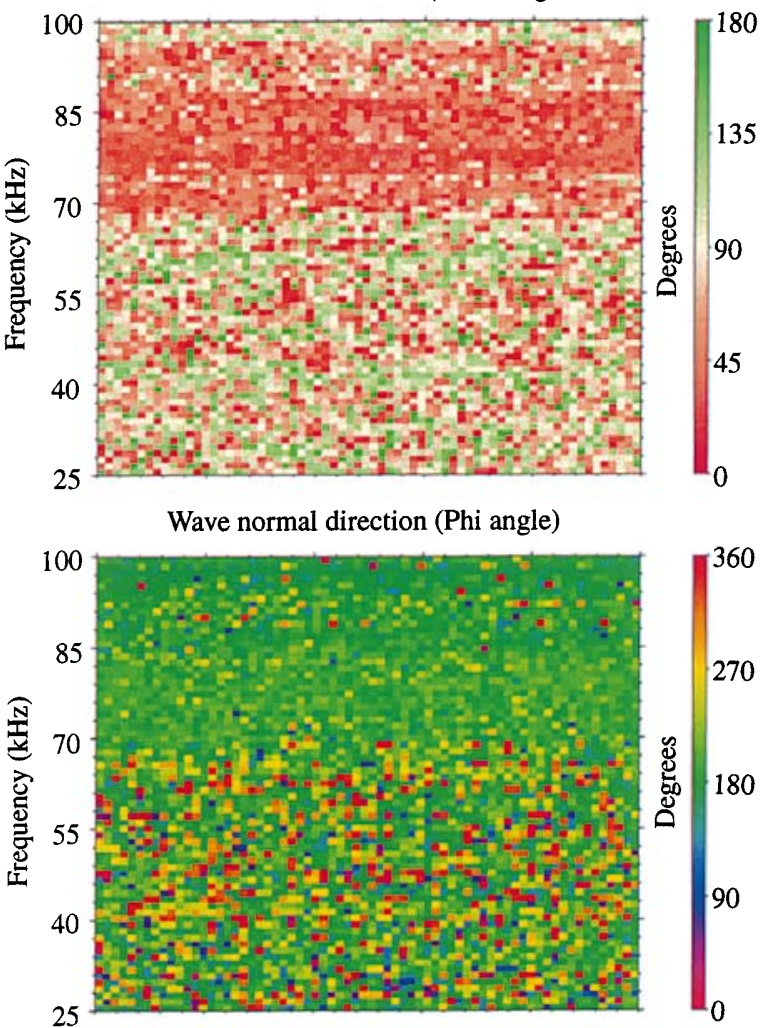


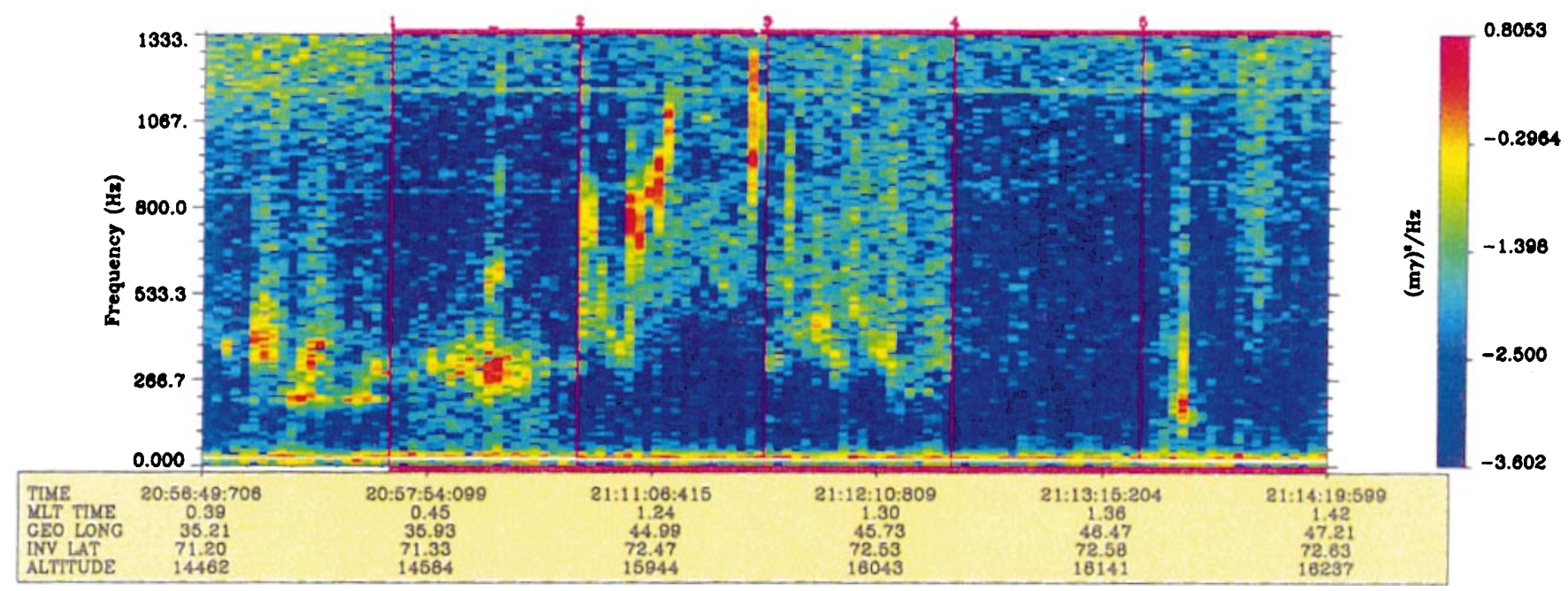

Fig. 10. Successive high resolution spectrograms of nightside burst of ELF electromagnetic emission (see text). The observation was made on 11 November, 1996

\subsection{Propagation characteristics of an AKR element}

A wave-normal analysis has been performed over the first $0.32 \mathrm{~s}$ of the AKR emission displayed on the bottom panel of Fig. 8 (or, which is the same, the top panel of Fig. 9). The AKR element of interest is included in the $70-85 \mathrm{kHz}$ frequency band. Outside of these band, and especially at the highest frequencies, the signal to noise ratio is too weak to allow a wave normal analysis. Again, due to uncertainties in the calibration function of the electric antennas, the units given are indicative only.

According to the length of the recording, wave normal analyses have been performed over several successive elementary time intervals. The results have been gathered on frequency/time diagrams. The highresolution auto-power spectra of $\mathrm{Bx}$ and $\mathrm{Ey}$ are displayed on the two upper panels of the left part of Fig. 9. The AKR element looks very stationary in time. For this case at least, no finer structure comes out when the time and frequency resolutions are increased. Except at the interference frequencies and at a few points in the frequency/time diagram, most estimates of the degree of polarization have values lower than 0.9. However, a simple examination of the right part of Fig. 8 shows that the waves are probably more coherent that one could expect from that. All the propagation parameters (ellipticity, $\theta$ and $\phi$ angles) have nearly constant values over the whole frequency band and the full time interval. There is practically no difference in the region where the degree of polarization is greater than 0.9 and outside. In the estimation theory, this means that the estimators we used are stable, and so that the statistical errors made on the estimated parameters are smaller than the statistical errors made on the calculation of the spectral matrix elements (see Eq. 1). Then, one can consider that the $\mathbf{k}$ vectors are contained in a more or less narrow cone with a stable central direction. Returning to the values of the propagation parameters, we observe: (1) that the polarization is quasi-circular (ellipticity value $\sim 0.9$ ); (2) that the waves are right polarized $\left(\theta<90^{\circ}\right)$, i.e., propagate in the $R$-X mode, with wave normals weakly oblique $\left(\sim 30^{\circ}\right)$; and (3) that the $\phi$ values are $\sim 180^{\circ}$. These results are not that different from those obtained in Sect. 3.2. The propagation characteristics of the (non-drifting) fine structure we have analyzed are the same as the one of structureless AKR emissions.

\section{Bursts of ELF electromagnetic emissions}

Several types of bursts of ELF emissions are detected on INTERBALL 2. Events of the "lion roar" type (Smith et al., 1969; Gurnett and Frank, 1972; Smith and Tsurutani, 1976) are observed on the night side. They are clearly electromagnetic. An example is given in the frequency/time diagram of Fig. 10. In that diagram, the Bx spectrogram of six successive sets of high-resolution data have been sticked altogether. Each one was estimated over a $2 \mathrm{~s}$ time interval. The time marks give an indication of the recording UT time. One observes bursts of a few seconds time duration, with frequency bandwiths of the order of $200 \mathrm{~Hz}$ and central frequencies which can vary up to $\sim 800 \mathrm{~Hz}$ in $1 \mathrm{~s}$. Although the signal energy is very weak on By and Bz, wave-normal directions may be estimated. A figure analogous to Figs. 5 and 7 has been drawn, but it is not represented here. By the way, the propagation characteristics can easily be guessed from a simple examination of the auto- and cross-power spectra. In the frequency band $\sim 260$ $340 \mathrm{~Hz}$, where the Bx auto-power spectra is maximum, the waves are both strongly polarized (degree of polarization $\sim 0.98$ ) and linearly polarized (ellipticity $\sim 0.1$ ). Their wave-normal vectors are perpendicular to the Earth's magnetic field $\left(\theta \sim 70-110^{\circ}\right)$. The sense of polarization can hardly be expressed in terms of $R$ or $L$ mode.

ELF emissions observed on the day side present quite different signatures in the frequency/time domain (see Fig. 11). They consist of bursts of a few minutes' 
INTERBALL-2, MEMO, Ey spectrogram, orbit 254, 29 October 96
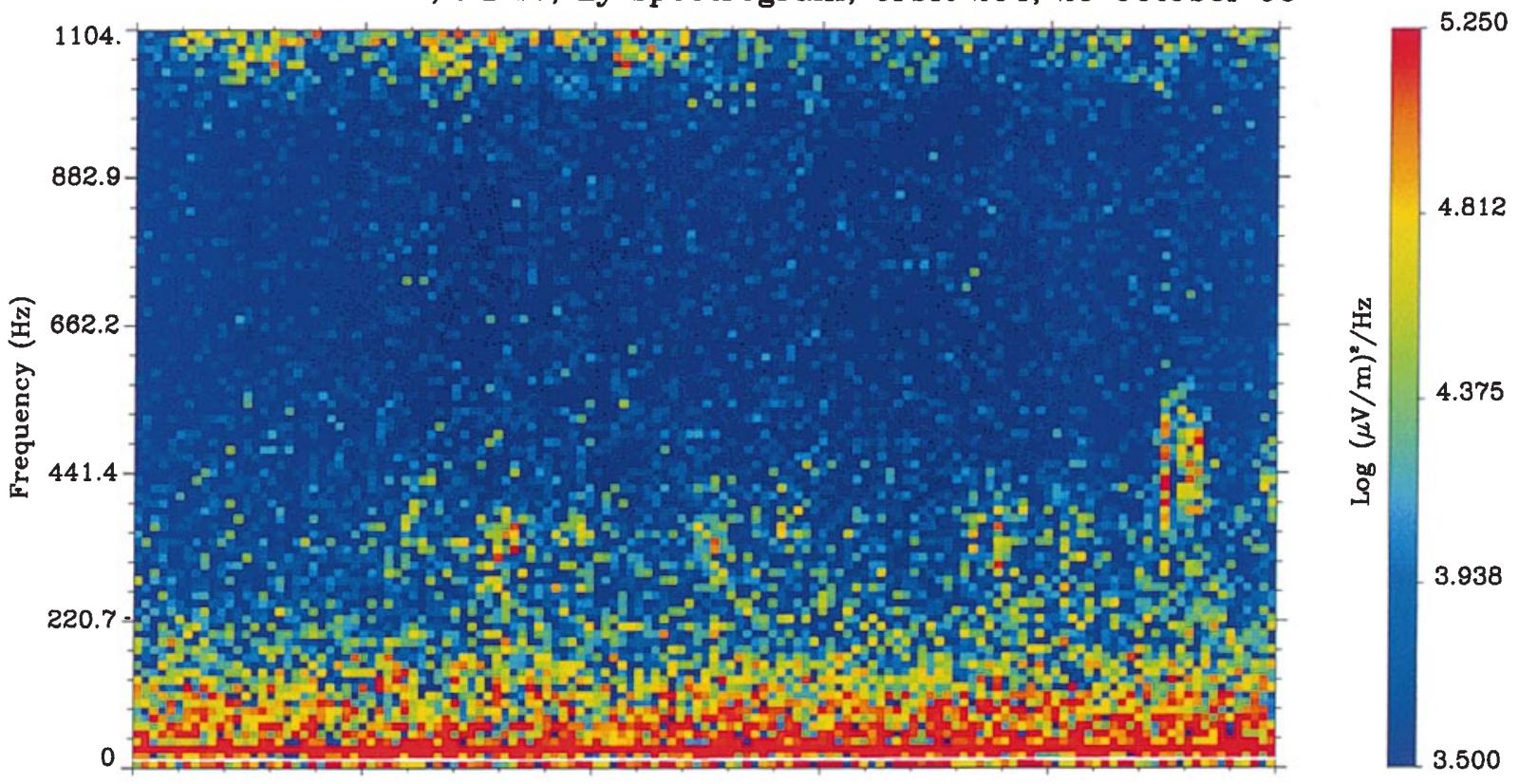

INTERBALL-2, MEMO, Bz spectrogram, orbit 254, 29 October 96

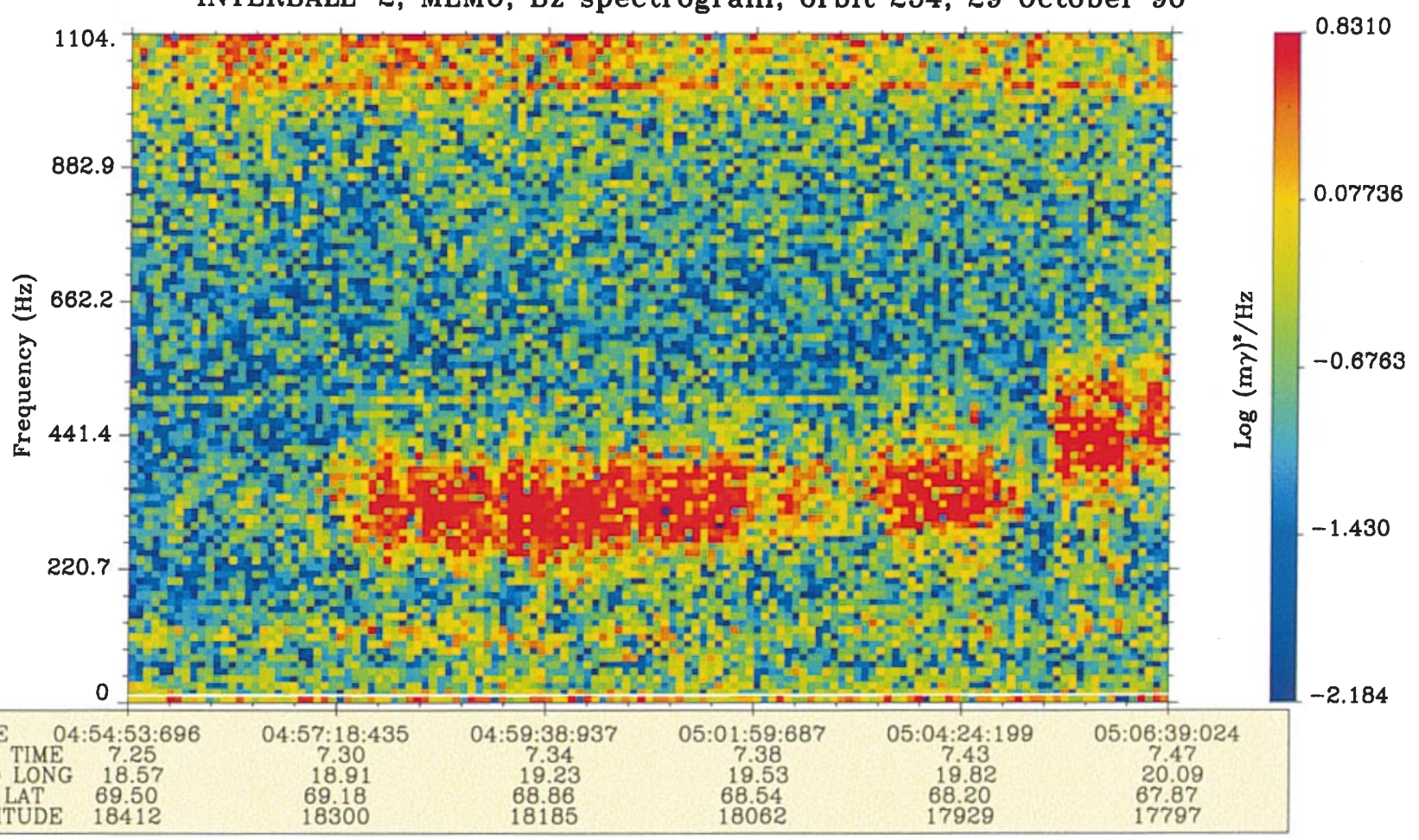

Fig. 11. Spectrogram of the Ey and Bx component for a dayside burst of ELF electromagnetic emission

duration, with an increase of the frequency band and/or of the central frequency when the invariant latitude is decreased. Most of the time they are detected on the magnetic antennas only (bottom panel of Fig. 11). However, in each 2 min approximately (i.e., each spin period), some energy is seen on the electric antennas (top panel). The lower cutoff frequency is of the order of 150 to $300 \mathrm{~Hz}$. The higher cutoff frequency, based on many examples, rarely exceeds $1 \mathrm{kHz}$. Statistics obtained from three months of MEMO functioning are obviously biased, but it should be noted that the emissions are detected each time measurements are made in one of the following two domains: (1) $08<\mathrm{MLT}<12,70<$ invariant latitude $<76^{\circ}$; (2) $04<$ MLT $<08,63^{\circ}<$ invariant 
latitude $<70^{\circ}$. An extension in Invariant latitude is possible for the first domain (we do not have any data at higher invariant latitudes in this MLT time interval). The boundaries are quite well defined for the second domain. Due to present problems with magnetometer and attitude data, the propagation characteristics have not been determined so far. Further work is needed to determine if the nightside and dayside phenomena have a similar origin or if they are quite distinct phenomena.

\section{Provisional conclusion}

The MEMO experiment is a complete wave experiment that is connected to a total of six electric and nine magnetic independent sensors. It provides waveforms associated with the measurement of two to five components in three frequency bands: ELF $(5-1000 \mathrm{~Hz})$, VLF $(1-20 \mathrm{kHz})$, LF $(20-250 \mathrm{kHz})$. Preliminary analyses have been focused on night time AKR and associated phenomena. The results are summarized as follows.

1. VLF hiss emissions are mainly whistler mode emissions. But other modes may be present. The lowest frequencies may be left polarized and the upper frequencies may propagate in the right polarized $\mathrm{Z}$ mode.

The local plasma frequency is accurately determined from the VLF emission when the $L=0$ low cutoff frequency is observed. Cases are found where the local plasma frequency so estimated is at the upper frequency of the VLF hiss emission and others where it is a few $\mathrm{kHz}$ below. When the $L=0$ low cutoff frequency is not observed, the local plasma frequency may be determined either from a natural resonance of the medium or for a transition in the power spectrum of the field components assuming it is a transition between the whistler mode and the right polarized $\mathrm{Z}$ mode. In a first approximation, density cavities may be characterized by low values of the low and high cutoff frequencies.

In the examples analyzed so far, the VLF hiss emissions cannot be associated with plane waves. Full wave normal analyses require the use of wave distribution function techniques (Storey and Lefeuvre, 1979, 1980; Lefeuvre et al., 1981; Lefeuvre and Helliwell, 1985; Santolik, 1995).

2. AKR emissions. Wave and particle data measured on INTERBALL 2 just above source regions present similarities with measurements performed by VIKING during source crossings: simultaneous observation of AKR, precipitating electrons, and upgoing ions; maximum of power and flux within local density cavities, presence of BEN and ELF turbulence. This suggest a ducted propagation from the source to the point of observation.

First direct estimations of the propagation characteristics of AKR, just above the source region, have shown the presence of two frequency bands with different polarization parameters. A low-frequency band $(120-170 \mathrm{kHz})$, with $R-\mathrm{X}$ mode waves circularly polarized propagating with averaged $\mathbf{k}$ vectors weakly oblique as regards to the Earth magnetic field direction.
A high-frequency band $(170-240 \mathrm{kHz})$, with $L$-O mode waves linearly polarized and propagating with $\mathbf{k}$ vectors practically along the Earth's magnetic field. This is consistent with generations models of AKR involving a spatial confinment of the waves within (and probably just above) the source region (Calvert, 1981, 1982; Louarn and Le Quéau, 1996a, b).

Basically, AKR fine structures observed on INTERBALL 2 are quite similar to the ones observed previously by other satellites. However, the detection of multiplets suggests the presence of a non-linear phenomena. Wave normal analyses performed over a (non-drifting) AKR element give results similar to the ones obtained for structurless emissions.

A new finding is the observation of an electrostatic ELF emission, not associated with any local ion gyrofrequency.

3. Bursts of ELF emissions. Nightside "lion roars" have been observed. They propagate with wave normals nearly perpendicular to the Earth's magnetic field direction. They are linearly polarized.

Dayside burst of ELF emissions are observed in welldefined MLT and invariant latitude domains. Up to now, it is not known if they have the same origin as lion roars or not.

Acknowledgements. The authors are very grateful to one referee for excellent comments and suggestions. They acknowledge valuable discussions on INTERBALL 2 data with Drs N. Dubouloz (HYPERBOLOID PI), Prof. J. Hanasz (POLRAD PI), S. Perraut (IESP PI) and J. A. Sauvaud (ION PI). They are grateful to Mr. J. $\mathrm{Y}$. Brochot who is presently in charge of the data handling of the MEMO data. They thank the LPCE technicians who contributed to the fabrication of the MEMO package (Messr. P. Badets, M. Chartier, J. P. Dumé, P. Fergeau, P. Fort†, J. Lebihan, T. Lemaire, M. Lévêque, P. Martin, J. M. Moreau, J. C. Toussaint ${ }^{\dagger}$, T. Vincent and $\mathrm{P}$. Zamora) and to the preparation of the data handling (C. Delannoy, J. Y. Brochot, Y. Prudhomme). They also thank the CNES members involved in the preparation of the INTERBALL mission (G. Azoulay, J. Durand, D. Fournier, J. C. Kosik, R. Marrama and J. P. Thouvenin). This work was financially supported by CNRS/SDU-INSU and CNES.

Topical Editor K.-H. Glassmeier thanks R. F. Benson for his help in evaluating this paper.

\section{References}

Baumback, M. M., and W. Calvert, The minimum bandwiths of auroral kilometric radiation, Geophys. Res. Lett., 14, 119, 1987.

Bahnsen, A., B. M. Pedersen, M. Jespersen, E. Ungstrup, L. Eliasson, J. S. Murphree, R. D. Elphinstone, B. Blomberg, G. Holmgren, and L. J. Zanetti, Viking observations at the source region of auroral kilometric radiation, J. Geophys. Res., 94, 6643-6654, 1989.

Benson, R. F., and W. Calvert, ISIS 1 observations at the source of auroral kilometric radiation, Geophys. Res. Lett., 6, 479-482, 1979

Benson R. F., M. M. Mellott, R. L. Huff, and D. A. Gurnett, Ordinary mode auroral kilometric radiation fine structure observed by DE 1, J. Geophys. Res., 93, 7515, 1988.

Berthelier, J. J., F. Lefeuvre, M. M. Mogilevsky, O. A. Molchanov, Y. I. Galperin, J. F. Karczewski, R. Ney, G. Gogly, C. Guérin, M. Leveque, J. M. Moreau, and F. X. Sené, Measurements of

\footnotetext{
$\dagger$ deceased
} 
the VLF electric and magnetic components of waves and DC electric field on board the AUREOL-3 spacecraft: the TBFONCH experiment, Ann. Géophysicae, 38, 643-667, 1982.

Born, M., and E. Wolf, Principle of optics, electromagnetic theory of propagation, interferences and diffraction of light, Sixth edn, Pergamon Press, 1980.

Calvert, W., The auroral plasma cavity, Geophys. Res. Lett., 8, 919-921, 1981.

Calvert, W., A feedback model for the source of auroral kilometric radiation, J. Geophys. Res., 87, 8199-8214, 1982.

Calvert, W., DE 1 measurements of AKR wave directions, Geophys. Res. Lett., 12, 381-384, 1985.

Calvert, W., and. K. Hashimoto, The magnetoionic modes and propagation properties of auroral radio emissions, J. Geophys. Res., 95, 3943-3957, 1990.

Delahaye, J. Y., J. Lavergnat, R. Ney, J. F. Karczewski, and F. X. Sené, Wave measurements in the ARAKS experiment, Space Sci. Instr., 4, 143-179, 1978.

de Feraudy, H., B. M. Pedersen, A. Bahnsen, and M. Jespersen, Viking observations of auroral kilometric radiation from the plasmasphere to night auroral oval source regions, Geophys. Res. Lett., 14, 511-514, 1987.

de Feraudy, H., A. Bahnsen, and M. Jesperen, Observation of nightside and dayside auroral kilometric radiation with Viking, in Proc. 2nd International Workshop on Radio Emission from Planetary Magnetosphere, Ed. H. O. Rucker, S. J. Bauer, and B. M. Pedersen, p. 239, Verlag der Österreichischen Akademie der Wissenschaften, Vienna, 1988.

Gurnett, D. A., and L. A. Frank, VLF hiss and related plasma observations in the polar magnetosphere, J. Geophys. Res., 77, 172-190, 1972.

Gurnett, D. A., and L. A. Frank, Plasma waves in the polar cusp: observations from Hawkeye 1, J. Geophys. Res., 83, 1447-1462, 1978.

Gurnett, D. A., R. R. Anderson, F. L. Scarf, R. W. Fredericks, and E. J. Smith, Initial results from the ISEE 1 and 2 plasma wave investigation, Space Sci. Rev., 23, 103, 1979.

Gurnett, D. A., and R. R. Anderson, The kilometric radio emission spectrum: Relationship to auroral acceleration processes, in Physics of Auroral Arc Formation, Geophys. Monogr. Ser., vol 25, Ed. S.-I. Akasofu and J. R. Kan, pp. 341-350, AGU, Washington, D.C., 1981.

Gurnett, D. A., S. D. Shawhan, and R. R. Shaw, Auroral hiss, Z mode radiation, and auroral kilometric radiation in the polar magnetosphere: DE 1 observations, J. Geophys. Res., 88, 329$340,1983$.

Hilgers, A., A. Roux, and R. Lundin, Characteristics of AKR sources: a statistical description, Geophys. Res. Lett., 18, 1493, 1991.

Hilgers, A., B. Holback, G. Holmgren, and R. Boström, Probe measurements of low plasma densities with applications to the auroral acceleration region and auroral kilometric radiation sources, J. Geophys. Res., 97, 8631, 1992.

Kimura, I., K. Hashimoto, I. Nagano, T. Okada, M. Yamamoto, T. Yoshino, H. Matsumoto, M. Ejiri, and K. Hayashi, VLF observations by the AKEBONO (EXOS-D) satellite, J. Geomagn. Geoelectr., 42, 459-478, 1990.

Kasahara, Y., K. Yoshida, T. Matsuo, I. Kimura, and T. Mukai, Propagation characteristics of auroral hiss observed by the Akebono satellite, J. Geomag. Geoelectr., 47, 509-525, 1995.

Krasnosel'skikh, V., and F. Lefeuvre, Strong Langmuir turbulence in the space plasmas, the problem of recognition, in Proc. ESA meeting on Spatio-temporal analysis for resolving plasma turbulence, ESA WPP-047, 237-242, Aussois, 1993.

Kravtchenko-Berejnoi, V., Utilisation des analyses polyspectrales dans l'étude des processus turbulents dans les plasmas spatiaux, Thèse de Doctorat, University of Orléans, 1994.

Kravtchenko-Berejnoi, V., F. Lefeuvre, V. Krasnosel'skikh, and D. Lagoutte, On the use of the fourth order spectral analysis to detect non-linear wave-wave interactions, Signal Proc., 42, 291309, 1995a.
Kravtchenko-Berejnoi, V., V. Krasnosel'skikh, D. Mourenas and F. Lefeuvre, Higher order spectra and analysis of a non-linear dynamic model, in Proc. CLUSTER workshop on data analysis tools, ESA SP-371, 61-68, 1995b.

Lagoutte, D., F. Lefeuvre, and H. Hanasz, Application of bicoherence analysis in study of wave interactions in space plasma, $J$. Geophys. Res., 94(A1), 435-442, 1989.

Lagoutte, D., and P. Latrémolière, SWAN 2.0 user's guide, LPCE/ NI/003.C - Part 1, Lab. Phys. Chime Environ., CNRS, Orléans, 1997a.

Lagoutte, D., and P. Latrémolière, SWAN 2.0 analysis tools, LPCE/NI/003.C - Part 2, Lab. Phys. Chime Environ., CNRS, Orléans, $1997 \mathrm{~b}$.

Lefeuvre, F., M. Parrot, and C. Delannoy, Wave distribution functions estimation of VLF electromagnetic waves observed on board GEOS 1, J. Geophys. Res., 86, 2359-2375, 1981.

Lefeuvre, F., T. Neubert and M. Parrot, wave normal directions and wave distribution functions for ground-based transmitter signals observed on GEOS 1, J. Geophys. Res., 87, 6203-6217, 1982.

Lefeuvre, F., and R. A. Helliwell, Characterization of the source of VLF hiss and chorus observed on-board GEOS 1, J. Geophys. Res., 90, 6419-6438, 1985.

Lefeuvre, F., Y. Marouan, M. Parrot and J. L. Rauch, Rapid determination of the sense of polarization and the sense of propagation for random electromagnetic fields, applications to GEOS 1 and AUREOL 3 data. Annales Geophysicae, 6, 457468, 1986. [Correction, Annales Geophysicae, 5A(4), 252, 1987].

Lefeuvre, F., M. Parrot, M. Mogilevsky, J. L. Rauch, B. Poirier, C. Delannoy, J. P. Dumé, P. Fergeau, M. Lévêque, P. Martin, J. M. Moreau, P. Zamora, Multicomponent measurements of waves on-board the auroral satellite of the INTERBALL project: the MEMO experiment, Kosm. Issled., to be published, 1998.

Louarn, P., Radio emissions from filamentary sources: a simple approach, in Planetary Radio Emissions IV, Ed. H. O. Rucker, S. J. Bauer, and A. Lecacheux, Verlag der Österreicchischen Akademie Der Wisseschaften, Wien, pp 153-165, 1996.

Louarn, P., and D. Le Quéau, Generation of the auroral kilometric radiation in plasma cavities: - I. Experimental study, Planet. Space Sci., 44, 199-210, 1996a.

Louarn, P., and D. Le Quéau, Generation of the auroral kilometric radiation in plasma cavities: - II. The cyclotron maser instability in small scale sources, Planet. Space Sci., 44, 211-224, 1996b.

Means, J. D., Use of the three-dimensional covariance matrix in analyzing the properties of plane waves, J. Geophys. Res., 77, 5551-5559, 1972.

Morioka, A., H. Oya, and S. Miyatake, Terrestrial kilometric radiation observed by the satellite Jikiken, J. Geomagn. Geoelectr., 33, 37, 1981.

Mourenas, D., V. V. Krasnosselskikh, and C. Béghin, Semirelativistic maser cyclotron instabilities in multicomponent plasmas, $J$. Geophys. Res., 98, A4, 5855, 1993.

Parrot, M., F. Lefeuvre and Y. Marouan, On the direct identification of propagation modes for HF plasma waves, J. Geophys. Res., 94, 17,049-17,062, 1989.

Pinçon, J. L., Y. Marouan, and F. Lefeuvre, Interpretation of measurements of the polarization percentage, Ann. Géophysicae., 10, 82-95, 1980.

Pottelette, R., M. Malingre, A. Bahnsen, and M. Jespersen, Observations of intense electrostatic hiss bands in the source regions of auroral kilometric radiation, Geophys. Res. Lett., 14, 515-518, 1987.

Pottelette, R., R. A. Treumann, and N. Dubouloz, Auroral kilometric radiation generation from lower hybrid solitons upper hybrid waves interaction, J. Geophys. Res., 97, A8, 12029, 1992.

Roux, A., A. Hilgers, H. De Feraudy, D. Le Quéau, P. Louarn, S. Perraut, A. Bahnsen, M. Jespersen, E. Ungstrup, M. André, Auroral kilometric radiation sources: in situ and remote observations from Viking, J. Geophys. Res., 98, 11,65711,670, 1993. 
Samson, J. C., Description of the polarization states of vector processes: application to ULF magnetic fields, J. R. Astron. Soc., 34, 403-419, 1973.

Samson, J. C., and J. V. Olson, Some comments of the descriptions of the polarization state of waves, Geophys. J. R. Astron. Soc., 61, 115-129, 1980.

Santolik, O., Etude de la fonction de distribution des ondes dans un plasma chaud, PHD Thesis, University of Orleans, 1995.

Shawhan, S. D, and, D. Gurnett, Polarization measurements of auroral kiloùetric radiation by Dynamics Explorer-1, Geophys. Res. Lett., 9, 913-916, 1982.

Smith, E. J., R. E. Holzer, and C. T. Russel, Magnetic emissions in the near magnetosheath at frequencies near $100 \mathrm{~Hz}, J$. Geophys. Res., 74, 3027, 1969.

Smith, E. J., and B. T. Tsurutani, Magnetosheath lion roars, J. Geophys. Res., 81, 2261, 1976.
Storey, L. R. O., and F. Lefeuvre, The analysis of 6-component measurements of a random electromagnetic wave field in a magnetoplasma. I. The direct problem. Geophys. J. R. Astron. Soc., 56, 255-269, 1979.

Storey, L. R. O., and F. Lefeuvre, The analysis of 6-component measurements of a random electromagnetic wave field in a magnetoplasma. II. The integration kernels. Geophys. J. R. Astron. Soc., 62, 173-194, 1980.

S-300 Experimenters, Measurements of electric and magnetic wave fields and of cold plasma parameters on-board GEOS-1. Preliminary results, Planet. Space Sci., 27, 317-339, 1979.

Ungstrup, E., A. Bahnsen, J. K., Wong, M. André and L. Matson, Energy source and generation mechanism for auroral kilometric radiation, J. Geophys. Res., 95, 5973, 1990.

Wu, C. S., and L. C. Lee, A theory of the terrestrial kilometric radiation, Astrophys. J., 230, 621-626, 1979. 